\title{
Comparative proteomic analysis uncovers potential biomarkers involved in the anticancer effect of Scutellarein in human gastric cancer cells
}

\author{
VENU VENKATARAME GOWDA SARALAMMA ${ }^{1,2}$, PREETHI VETRIVEL ${ }^{1}$, HO JEONG LEE ${ }^{3}$, SEONG MIN KIM $^{1}$, \\ SANG EUN HA ${ }^{1}$, RAJESWARI MURUGESAN ${ }^{4}$, EUN HEE KIM ${ }^{5}$, JEONG DOO HEO ${ }^{3}$ and GON SUP KIM ${ }^{1}$ \\ ${ }^{1}$ Research Institute of Life Science and College of Veterinary Medicine, Gyeongsang National University, Jinju, \\ Gyeongnam 52828; ${ }^{2}$ College of Pharmacy, Yonsei University, Incheon 21983; ${ }^{3}$ Gyeongnam Department of \\ Environment Toxicology and Chemistry, Biological Resources Research Group, Korea Institute of Toxicology, \\ Jinju, Gyeongnam 52834, Republic of Korea; ${ }^{4}$ Department of Biochemistry, Biotechnology and Bioinformatics, \\ Avinashilingam Institute for Home Science and Higher Education for Women, Coimbatore, Tamil Nadu 641043, India; \\ ${ }^{5}$ Department of Nursing Science, International University of Korea, Jinju, Gyeongnam 52833, Republic of Korea
}

Received December 19, 2019; Accepted May 28, 2020

DOI: $10.3892 /$ or.2020.7677

\begin{abstract}
Scutellarein (SCU), a flavone that belongs to the flavonoid family and abundantly present in Scutellaria baicalensis a flowering plant in the family Lamiaceae, has been reported to exhibit anticancer effects in several cancer cell lines including gastric cancer (GC). Although our previous study documented the mechanisms of Scutellarein-induced cytotoxic effects, the literature shows that the proteomic changes that are associated with the cellular response to SCU have been poorly understood. To avoid adverse side-effects and significant toxicity of chemotherapy in patients who react poorly, biomarkers anticipating therapeutic responses are imperative. In the present study, we utilized a comparative proteomic analysis to identify proteins associated with Scutellarein (SCU)-induced cell death in GC cells (AGS and SNU484), by integrating two-dimensional gel electrophoresis (2-DE), mass spectrometry (MS), and bioinformatics to analyze the proteins. Proteomic analysis between SCU-treated and DMSO (control) samples successfully identified 41 (AGS) and 31 (SNU484) proteins by MALDI-TOF/MS analysis and protein database search. Comparative proteomics analysis between AGS and SNU484 cells treated with SCU revealed a total of 7 protein identities commonly expressed and western blot analysis validated a subset of identified critical proteins, which were consistent with those of the 2-DE outcome. Molecular docking
\end{abstract}

Correspondence to: Professor Gon Sup Kim, Research Institute of Life Science and College of Veterinary Medicine, Gyeongsang National University, 501 Jinju-daero, Jinju, Gyeongnam 52828, Republic of Korea

E-mail: gonskim@gnu.ac.kr

Key words: biomarkers, gastric cancer, scutellarein, two-dimensional gel electrophoresis, CIP2A, PIK3CB studies also confirmed the binding affinity of SCU towards these critical proteins. Phosphatidylinositol 4,5-bisphosphate 3 -kinase catalytic subunit $\beta$ isoform (PIK3CB) protein expression was accompanied by a distinct group of cellular functions, including cell growth, and proliferation. Cancerous inhibitor of protein phosphatase $2 \mathrm{~A}$ (CIP2A), is one of the oncogenic molecules that have been shown to promote tumor growth and resistance to apoptosis and senescence-inducing therapies. In the present study, both PIK3CB and CIP2A proteins were downregulated in SCU-treated cells, which boosts our previous results of SCU to induce apoptosis and inhibits GC cell growth by regulating these critical proteins. The comparative proteomic analysis has yielded candidate biomarkers of response to SCU treatment in GC cell models and further validation of these biomarkers will help the future clinical development of SCU as a novel therapeutic drug.

\section{Introduction}

Gastric cancer was the fifth most prevalent malignancy in 2018 globally with an estimated 1 million new cases. Korea has been listed first among the global GC cases, with stomach cancer incidence rates in men of approximately 58 per 100,000 and in women of 24 per every 100,000, which is an unfortunate reality for many Koreans (1). Epigenetic alterations, aberrant molecular signaling pathways, and multiple genetic mutations are engaged in the development of GC (2). Substantial advances in diagnostic techniques, chemotherapy and surgical approaches, treatment by multidisciplinary teams and the advancement of novel therapeutic agents have shown minor improvement in survival rate in recent times (3). If we can identify the biomarkers of drug treatment responses and their efficacy, the unnecessary burden of adverse effects and noticeable toxicity from chemotherapy on GC patients unlikely to respond can be averted (4). It is imperative to identify the specific biomarkers and unique molecular patterns of the tumor to develop treatments that target the distinct tumor behavior. In the standard 
treatment of GC, a significant number of novel anticancer agents targeting dysfunctional molecular signaling pathways has been reported already, whereas others remain elusive $(5,6)$. The global proteomic approach is one of the computational technologies that have been improved in recent times over many other complementary techniques that briskly change our approach to cancer research (7). This empowers scientists to screen substantial numbers of proteins within clinical discrete samples, as well as different cell lines treated with specific drugs that help to analyze and confirm drug targets, find new disease biomarkers, design more adequate drugs, and estimate drug efficacy (8). The conventional proteomics approach was achieved by implementing a combination of 2-DE coupled with differential image analysis and protein identification using mass spectrometry (MALDI-TOF; matrix-assisted laser desorption/ ionization-time of flight) and bioinformatics to predominantly identify and characterize proteins in the tissues and cells from both in-vitro and in-vivo models $(8,9)$. Protein network, functional interpretation, and pathway analysis tools can help to address the difficulties in the illustration of the obtained proteomics data. To identify the activated pathway element of functional proteomic data, the analysis of proteomic data at the pathway level has become universally popular (10). The comparative proteomic analysis could persuade the molecular characterization of cellular events correlated with cancer developmental, signaling, and progression phases that leads to the discovery of cancer-specific protein markers, which provides the basis for understanding cancer progression, carcinogenesis and targets of protein molecules for anticancer agents (11).

Herbal products and their components have been identified as exhibiting anticancer effects by targeting dysregulated genes that contribute to carcinogenesis in several cancer cell lines by multiple cell signaling pathways $(12,13)$. Flavonoids are natural polyphenolic compounds that are abundantly present in plant parts, especially in leaves and fruits, and previous studies have demonstrated several anticancer effects by regulating multiple cellular mechanisms such as the PI3K/AKT/mTOR signaling pathway $(13,14)$. Fig. 1A shows Scutellarein (SCU), a flavone, which belongs to the family of flavonoids, that are abundantly present in perpetual herbs, such as Scutellaria barbata and Scutellaria baicalensis. Studies have demonstrated that SCU monomers, as well as SCU-containing flavonoid extracts, cover an immense range of biological applications, including anti-inflammatory, antioxidant, and anticancer activity, by regulating different biological activities (15). Our previous study demonstrated that SCU induces apoptotic cell death and inhibits cell proliferation via downregulation of MDM2 protein expression which in turns activated the tumor-suppressor protein p53; and the family of inhibitors of apoptosis proteins (cIAP1, cIAP2, and XIAP) were downregulated leading to caspase-dependent apoptosis in human GC cells (16). However, despite elucidation of the anticancer effect of SCU on GC cells, the global proteomic changes that encompass the cellular response to this compound have remained elusive. In the present study, we aimed to identify the novel protein biomarkers and characterize the protein alterations that can predict the treatment efficacy of SCU in GC cells. The comparative proteomic analysis may facilitate future molecular research on the anticancer effects of flavonoids.

\section{Materials and methods}

Cell lines, reagents and antibodies. Human GC cell lines, AGS and SNU484, were procured from the Korea Cell Line Bank (Seoul, Korea). Antibiotics (penicillin/streptomycin), fetal bovine serum (FBS) and RPMI-1640 growth medium were purchased from Gibco, Thermo Fisher Scientific, Inc. Compound Scutellarein was procured from Chengdu Biopurify Phytochemicals Ltd. (product no. 611130). Chemicals and materials utilized for electrophoresis were procured from Bio-Rad Laboratories, Inc. 3-(4,5-Dimethylthiazol-2-yl)-2,5-diphenyltetrazolium bromide (MTT) was purchased from Duchefa Biochemie. Antibodies for CIP2A (\#148053) and PI3KCB (\#3011S) were purchased from Cell Signaling Technology, Inc. OFD1 (\#ab222837), VCL (\#ab129002), and HIP1R (\#ab226197) antibodies were purchased from Abcam. The $\beta$-actin (\#MABT825) antibody was purchased from Millipore.

Treatment of SCU and determination of cell viability. Mycoplasma-free AGS and SNU484 cells were cultured in RPMI-1640 medium containing 10\% FBS (heat activated) and $1 \%$ antibiotics (penicillin/streptomycin) in an incubator with $5 \% \mathrm{CO}_{2}$ at $37^{\circ} \mathrm{C}$ in a humidified condition. To confirm mycoplasma contamination, we used the e-Myco ${ }^{\mathrm{TM}}$ Mycoplasma PCR Detection kit (iNtRON Biotechnology). Cell viability assay was performed using MTT assay. The cells were at a density of $5 \times 10^{5}$ cells per well in 24-well plates and grown overnight. Subsequently treat the cells were treated with the indicated concentrations of $\operatorname{SCU}(0,25,50,75$, and $100 \mu \mathrm{M})$ for $24 \mathrm{~h}$ at $37^{\circ} \mathrm{C}$. An amount $50 \mu \mathrm{l}$ of MTT solution $(0.5 \mathrm{mg} / \mathrm{ml})$ was added to each well after $24 \mathrm{~h}$, and incubation was carried out at $37^{\circ} \mathrm{C}$ for $3 \mathrm{~h}$ in a dark condition. DMSO $(300 \mu \mathrm{l})$ was added to each well to solubilize the formazan contained in the cells, and the absorbance was measured at $540 \mathrm{~nm}$ using a microplate reader (BioTek Instruments). Cell viability was expressed as a percentage of the control and experiments were conducted in triplicates for each assay condition.

Protein sample preparation for 2-DE analysis. AGS and SNU484 cells were treated or untreated (control) with SCU (75 $\mu \mathrm{M}$ ) for $24 \mathrm{~h}$. Whole proteins were extracted from both groups of cells as previously described (17). Briefly, after incubation time, the cells were harvested and lysed with lysis buffer on ice for $1 \mathrm{~h}$. The cell lysates were centrifuged at 20,000 x g at $4^{\circ} \mathrm{C}$ for $15 \mathrm{~min}$ and the supernatants were collected. Proteins were precipitated and extracted using the TCA precipitation method, followed by dissolving the lyophilized protein with $200 \mu \mathrm{l}$ of sample buffer for further analysis. The proteins were quantified according to the manufacturer's instructions using a BCA protein assay (Pierce ${ }^{\mathrm{TM}}$; Thermo Fisher Scientific, Inc.), and the protein samples were stored in a $-80^{\circ} \mathrm{C}$ freezer.

Two-dimensional gel electrophoresis. Proteins were separated using 2-DE, as reported in our previous study (17). Briefly, $400 \mu \mathrm{g}$ of protein per sample (equal quantity) were immobilized onto $18 \mathrm{~cm}$ ( $\mathrm{pH} \mathrm{4-7)}$ DryStrip Gels (GE Healthcare Immobiline ${ }^{\mathrm{TM}}$; Amersham Biosciences) for the first dimensional electrophoresis (isoelectric focusing: IEF) using Ettan DALT II system (Amersham Biosciences). After the 


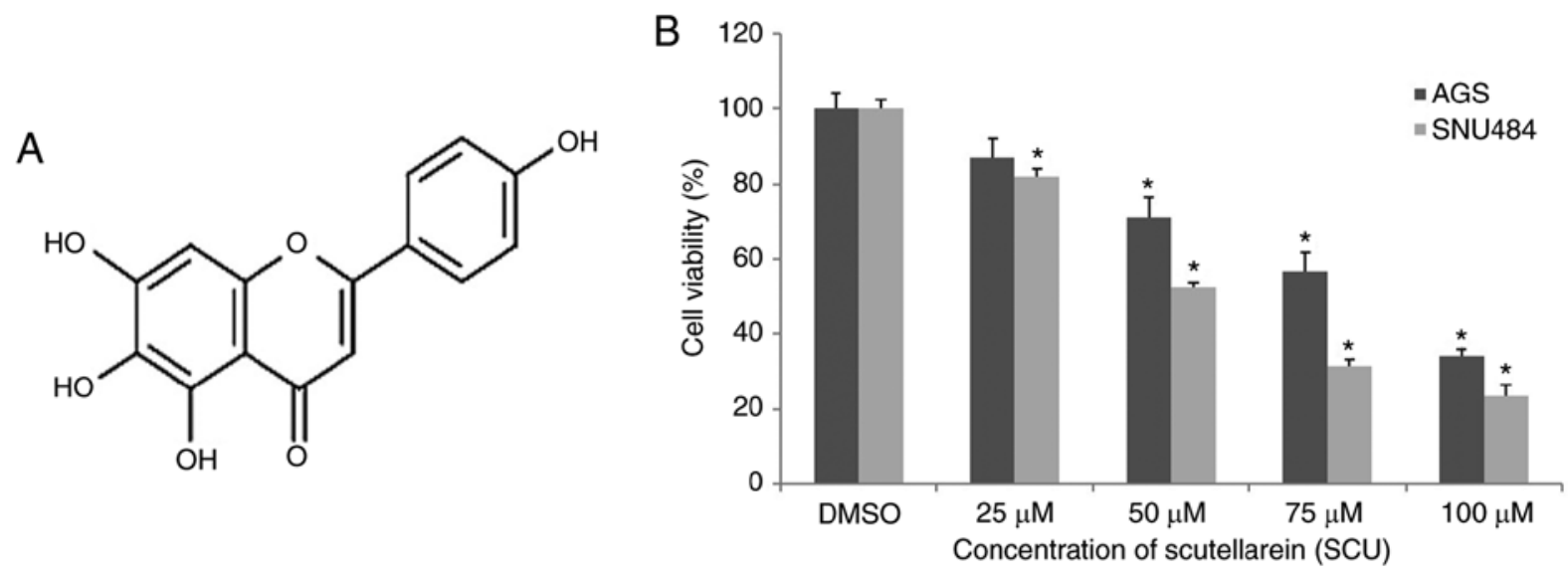

Figure 1. SCU attenuates GC cell viability in a dose-dependent manner. (A) Chemical structure of Scutellarein (5,6,7,4'-tetrahydroxy flavone). (B) AGS and SNU484 GC cells were treated with different concentrations of SCU (0-100 $\mu \mathrm{M})$ or untreated (DMSO) for $24 \mathrm{~h}$ followed determination of cell viability using MTT assay. The results are the representatives of three independent experiments and are expressed as mean \pm standard deviation (SD). Statistical differences were analyzed with Student's t-test and a one-way ANOVA test was implemented followed by Tukey's test for the comparison of multiple independent variables. ${ }^{*} \mathrm{P}<0.05$, significant difference vs. the control (DMSO). GC, gastric cancer; SCU, Scutellarein.

isoelectric focusing, the strips were equilibrated twice; first time with equilibration buffer containing $10 \mathrm{mg} / \mathrm{ml}$ dithiothreitol (DTT) and the second time with equilibration buffer containing $40 \mathrm{mg} / \mathrm{ml}$ iodoacetamide (IAA) for $15 \mathrm{~min}$ each. Proteins in equilibrated strips were then separated, depending upon the molecular weight, with the second dimension on $12 \%$ sodium dodecyl sulfate-polyacrylamide gel electrophoresis (SDS-PAGE). Silver nitrate staining methods were implemented as reported previously (18) with slight changes, and gels were prepared in triplicates for each assay condition. The silver-stained gels were scanned for image analysis, and Progenesis Samespots software version 4.0 Nonlinear Dynamics Ltd.) was used to perform spot density-based image analysis. Those spots were considered for further analysis depending on the difference in the spot intensities with fold-change $\geq 1.5$ and statistical significance of $\mathrm{P}<0.05$ in SCU-treated AGS and SNU484 cells, compared with the untreated (DMSO) groups.

Trypsin digestion and mass spectrometry analysis. Differentially expressed protein spots from the 2-DE gel were excised manually, and digestion of protein and MS analysis were performed as previously reported (19) with slight changes. Briefly, the selected gel spots were trypsin digested (Promega Corp.) for mass spectrometry analysis with 10-20 $\mu \mathrm{l}$ on ice for $45 \mathrm{~min}$. After incubation on ice, 10-20 $\mu \mathrm{l}$ of trypsin digestion buffer without trypsin was added, and digestion was carried out overnight at $37^{\circ} \mathrm{C}$. Proteins were extracted by adding 10-20 $\mu \mathrm{l}$ of the extraction buffer and incubated for $30 \mathrm{~min}$ at room temperature (RT) twice, and the solution was collected onto siliconized e-tubes. Collected extracts were lyophilized in a vacuum lyophilizer, and the pellets were re-suspended in a mixture of $1 \mu \mathrm{l}$ of extraction buffer and $1 \mu \mathrm{l}$ of matrix solution containing $\alpha$-acyano-4-hydroxycinnamic acid (HCCA). The suspension of matrix solution containing protein was targeted onto a MALDI-TOF plate, and the targeted spots were read by mass spectrometer (Voyager-DE STR; Applied Biosystems), supplied with delay ion extraction. Mass spectra were selected over a mass range of $\geq 3,000 \mathrm{Da}$.
Identification of protein from the MS data. To identify the proteins from the MS data, the peptide protein mass fingerprinting data were adopted to search against NCBI non-redundant protein database using the ProteinProspector (v 5.22.1) (http://www.prospector.ucsf.edu) peptide mass fingerprinting (PMF) data (20), and the SwissProt database (SwissPort.2017.11.01) was implemented to find the matching proteins, as defined previously 38 (17). Database search was performed using the following parameters: Homo sapiens (human) was used in terms of Taxonomy, trypsin with 1 missed cleavage permitted was used for digest specificity, peptide tolerance of less than $100 \mathrm{ppm}$ was used for fragment ions, carbamidomethyl (C) was used with fixed modifications and oxidation $(\mathrm{M})$ was used as a variable modification. Protein MOWSE scores $(\mathrm{P}<0.05)$ were considered statically significant.

Protein validation by immunoblotting. For western blotting, both cell lines were cultured in 6-well plates at $3 \times 10^{6}$ cells per well and after the cells reached optimal confluence, both the cell lines were treated with SCU $(75 \mu \mathrm{M})$ or untreated (DMSO) for $24 \mathrm{~h}$. Cells were harvested after incubation, and lysed in ice-cold RIPA buffer containing protease and phosphatase inhibitor. Total proteins were quantified using BCA protein assay and $15 \mu \mathrm{g}$ of proteins from each group were separated by $10-12 \%$ SDS-PAGE, and the protein bands were transferred onto a polyvinylidene difluoride (PVDF) membrane. The membranes were blocked with 5\% non-fat skim milk or BSA in Tris-buffered saline containing $1 \%$ Tween 20 (TBS-T, pH 7.4) at room temperature (RT) for $1 \mathrm{~h}$, and incubated overnight at $4^{\circ} \mathrm{C}$ at a 1:1,000 dilution of the respected primary antibody. The membranes were washed five times with TBS-T for 10 min each at RT, and incubated with a 1:2,000 dilution of HRP-conjugated secondary antibody for $3 \mathrm{~h}$ at RT. The membranes were then rewashed five times with TBS-T. Blots were developed using the ECL detection system (GE Healthcare Life Science). The bands were quantitatively analyzed using the ImageJ software version 1.52a (National Institutes of Health) (http://rsb.info.nih.gov). The densitometry 
readings of the bands were normalized according to $\beta$-actin expression.

Molecular docking analysis. In the present study, the binding affinity of Scutellarein was evaluated through macromolecular docking studies using Glide of Schrödinger-Maestro v.8.5 (in silico analysis) (21). Initially, the structure of the Scutellarein molecule was obtained and energy was minimized. The 3D structure of protein targets was downloaded from the PDB database (https://www.rcsb.org/). During docking, the receptor grids were determined for processed proteins, such that various ligand poses bind within the anticipated active site. Final scoring was performed on energy-minimized poses and displayed as Glide score. The best ligand binding pose with the least Glide/IFD score or energy was chosen. For each ligand, the best-docked pose with lowest Glide score value was recorded. Binding energy was calculated by implementing Schrödinger Prime based on molecular mechanics generalized born surface area (MM-GBSA). The interacting amino acids of the protein with the structure of SCU were viewed by the ligand-interaction diagram constructed by LigPlot.

Gene Ontology (GO) and pathway enrichment analysis. SwissProt identified proteins were further submitted to Web Gestalt (http://www.webgestalt.org), to find the GO annotations of the acquired differential proteins expressed in AGS and SNU cells treated with SCU independently. Emerging annotation were encapsulated based on the GOSlim set enrichments using a GOSlim Viewer to allocate their biological process, cellular component, and molecular function. The biological process identified from Web Gestalt was further studied individually for each protein annotations. A web-based tool GeneCodis (http://genecodis.cnb.csic.es) was used for comparative protein analysis between AGS and SNU484 cells treated with SCU (22). The significantly identified proteins were subjected to pathway analysis by utilizing the PANTHER (Protein Analysis Through Evolutionary Relationships, version 9.0) database (http://www.pantherdb.org ) in both the cell lines (23). STRING database (https://string-db.org) was used to identify protein-protein interaction among the differentially expressed protein as well as for the construction of individual protein clusters of commonly expressed proteins (24).

Statistical analysis. Statistical analysis was performed with the Student's t-test using SPSS version 10.0 for Windows (SPSS, Inc.) and a one-way ANOVA test was implemented followed by Tukey's test for the comparison of multiple independent variables. A fold-change $\geq 1.5$ and statistical significance of $\mathrm{P}<0.05$ were considered for the selection of differential expressed protein spots. Data were considered statistically significant at $\mathrm{P}<0.05$. All the results are expressed as the mean \pm standard deviation (SD) of triplicates.

\section{Results}

Scutellarein (SCU) inhibits the cell viability of GC cells. In our previous study, we reported that SCU was able to inhibit the cell viability of AGS and SNU484 human GC cells via inducing apoptotic cell death (16). Cell viability assay was performed after treatment with different concentrations of
$\operatorname{SCU}(0,25,50,75$ and $100 \mu \mathrm{M})$ for $24 \mathrm{~h}$, as shown in Fig. $1 \mathrm{~B}$ in AGS and SNU484 cells using MTT assay. SCU significantly inhibited the cell viability in a dose-dependent manner in both cell lines, when compared with the untreated control (DMSO only) group of cells. The $\mathrm{IC}_{50}(50 \%$ inhibitory concentration) value obtained for AGS cells was $62.88 \mu \mathrm{M}$ and for SNU484 cells was $59.45 \mu \mathrm{M}$. Henceforth for the subsequent experiments, we used 0 (control) and $75 \mu \mathrm{M}$ (Test) (most effective concentration) of SCU on both the GC cell lines.

Protein identification by 2-DE and MALDI/TOF-MS analysis. Proteomic analysis of AGS and SNU484 cells in response to SCU treatment was conducted. To analyze the proteome changes upon treatment with SCU to induce cell death in AGS and SNU484 cells, $400 \mu \mathrm{g}$ total proteins were separated by isoelectric focusing (IEF) on $18 \mathrm{~cm}$ IPG strips in the first dimension, and resolved by 2-DE, followed by silver staining for visualization. Three gels per sample were analyzed simultaneously by confirming the representative 2-DE patterns of protein spots from the control and SCU-treated (75 $\mu \mathrm{M})$ AGS and SNU484 cells (Figs. 2A and B and 3A and B respectively). After automatic spot identification, background subtraction, and volume normalization in AGS and SNU484 cells, a total of 58 and 43 spots were found to be differentially expressed between the untreated and SCU-treated cells (fold change $\geq 1.5$; $\mathrm{P}<0.05$ ) by using Progenesis Samespots image analysis software, respectively. After MALDI-TOF-MS, 41 (AGS) and 31 (SNU484) proteins were successfully identified upon database search (http://www.prospector.ucsf.edu). Among the identified proteins, 17 proteins were upregulated and 24 proteins were downregulated in the AGS cells treated with SCU, compared with the untreated group of cells and 18 proteins were upregulated and 13 proteins were downregulated in SNU484 cells treated with SCU compared with the untreated group of cells. Tables I (AGS) and II (SNU484) show the characterization of the identified proteins with their respective accession number, fold change, analytical isoelectric point, analytical molecular weight, and MOWSE score, and the sequence coverage and number of peptide matches, respectively.

Comparative analysis of the functional enrichment of the differentially expressed proteins. Oral-facial-digital syndrome 1 protein (OFD1), vinculin (VINC), voltage-dependent calcium channel subunit $\alpha-2 / \delta-1$ (CACNA2D1), Huntingtin-interacting protein 1-related protein (HIP1R), proto-oncogene vav 1 (VAV1), phosphatidylinositol 4,5-bisphosphate 3-kinase catalytic subunit $\beta$ isoform (PIK3CB) and synaptonemal complex protein 1 (SYCP1) were found to be the 7 proteins commonly expressed in both GC cells treated with SCU by the comparative analysis using GeneCodis (http://genecodis. cnb.csic.es) a web-based tool. The remaining 34 from AGS and 24 from SNU484 proteins were uniquely differentially expressed when treated with SCU as shown in Fig. 4. OFD1, VINC, HIP1R, and PIK3CB were downregulated in both cell lines, whereas CA2D1, VAV1 and SYCP1 protein expression were elevated in both cell lines treated with SCU compared with the control group of cells. Fig. S1 shows the protein clusters of the individual proteins (A) OFD1, (B) VINC, (C) HIPR1, (D) CA2D1, (E) PIK3CB, (F) VAV1 and (G) CIP2A by STRING database. Table IV (AGS) and Table V (SNU) show 
A

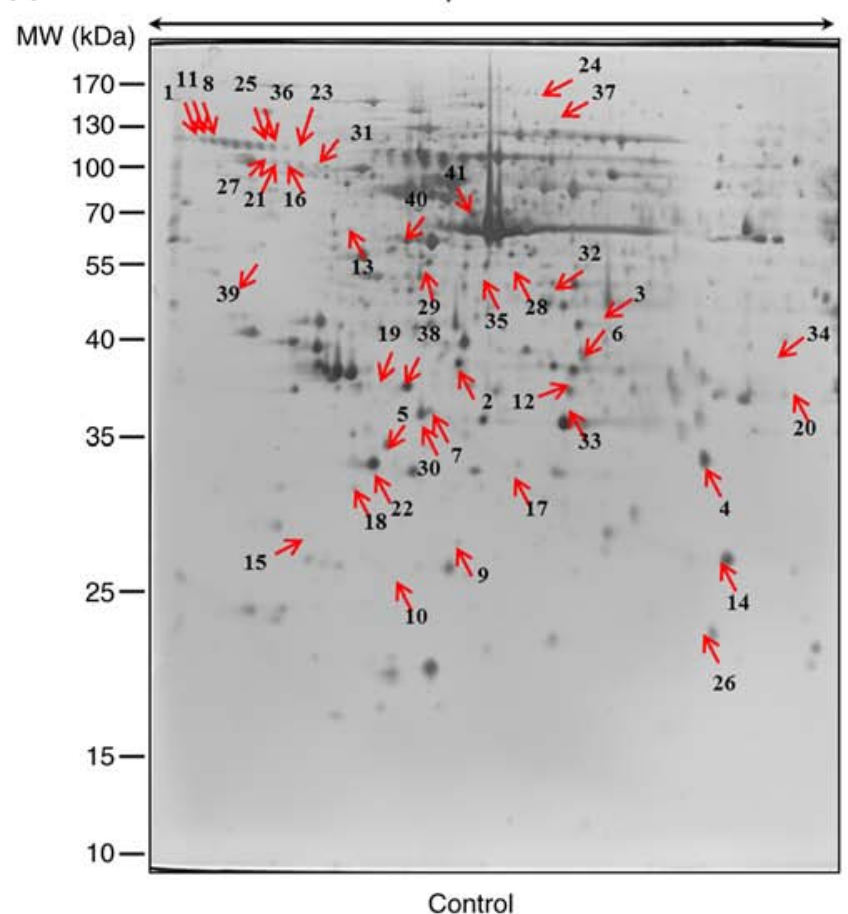

B

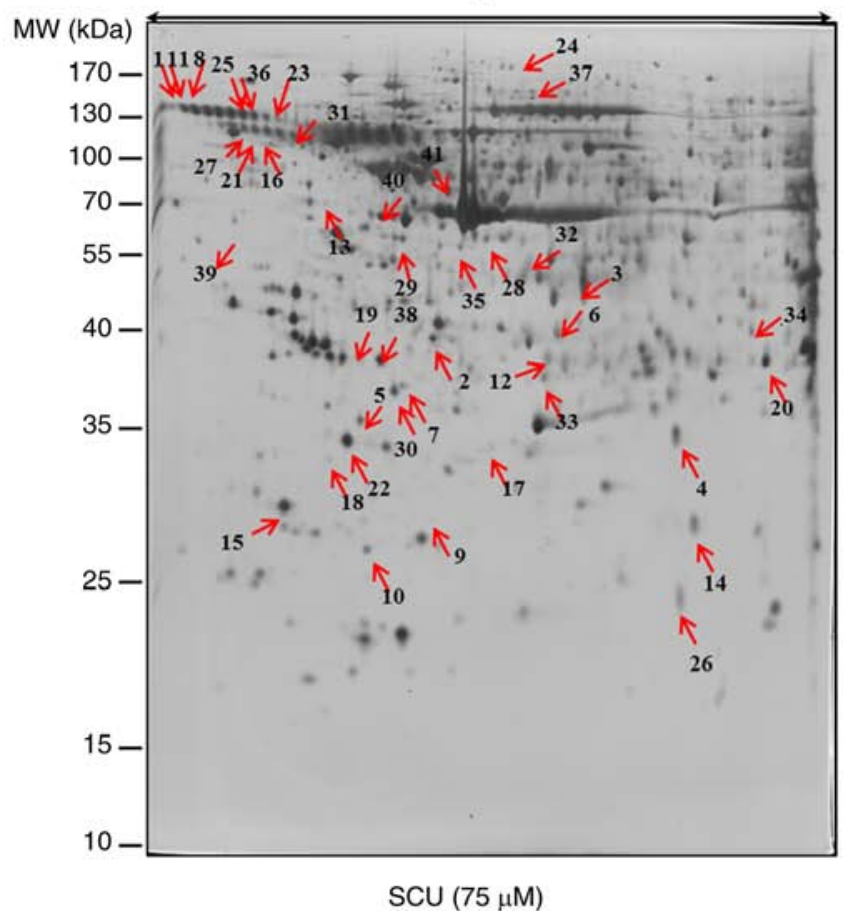

Figure 2. 2-DE protein pattern of differentially expressed proteins identified by MALDI-TOF-MS analysis in AGS cells. (A) Control (DMSO) and (B) SCU-treated $(75 \mu \mathrm{M}) \mathrm{GC}$ AGS cells. Cells were treated with the indicated concentrations of SCU or DMSO for $24 \mathrm{~h}$. A total of $400 \mu \mathrm{g}$ of total proteins was separated on 18-cm linear IPG strips ( $\mathrm{pH}$ 4.0-7.0) by IEF and 12\% SDS-PAGE gels were used in the second dimension of separation followed by silver staining of the gels. The arrows indicated by numbers are the protein spots identified successfully by MALDI-TOF-MS on protein database search. The experiments were performed in triplicate. 2-DE, two-dimensional gel electrophoresis; MALDI-TOF, matrix-assisted laser desorption/ionization-time of flight; IEF, isoelectric focusing; GC, gastric cancer; SCU, Scutellarein; MW, molecular weight.

A

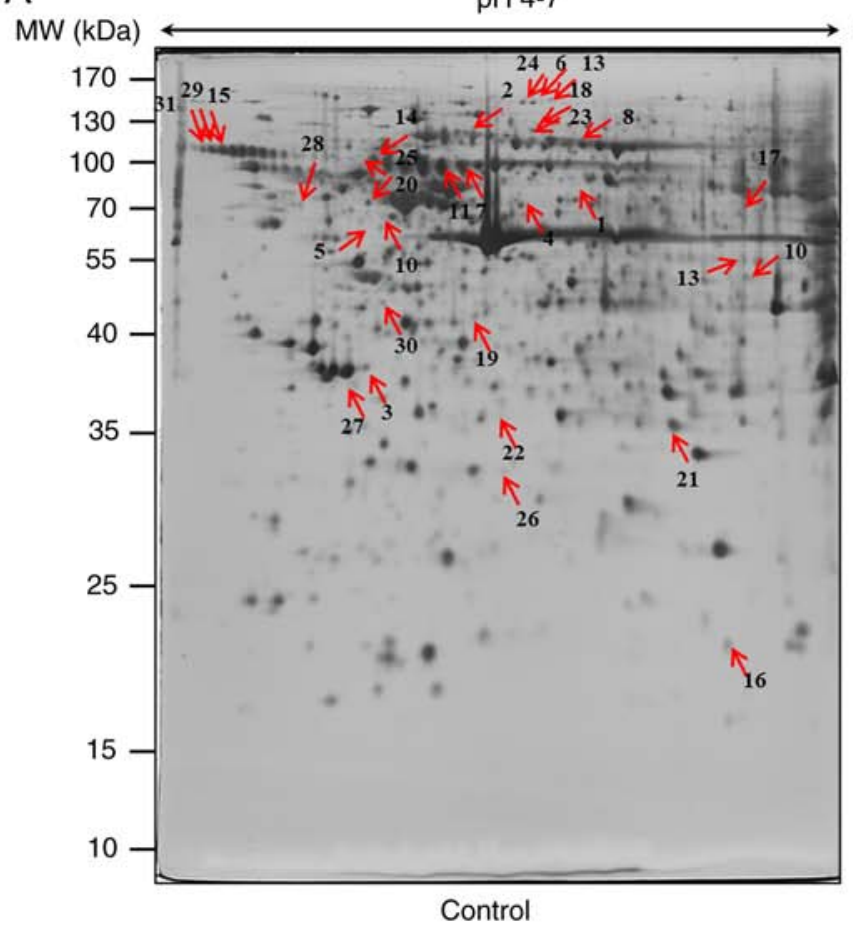

B $\mathrm{MW}(\mathrm{kDa})$

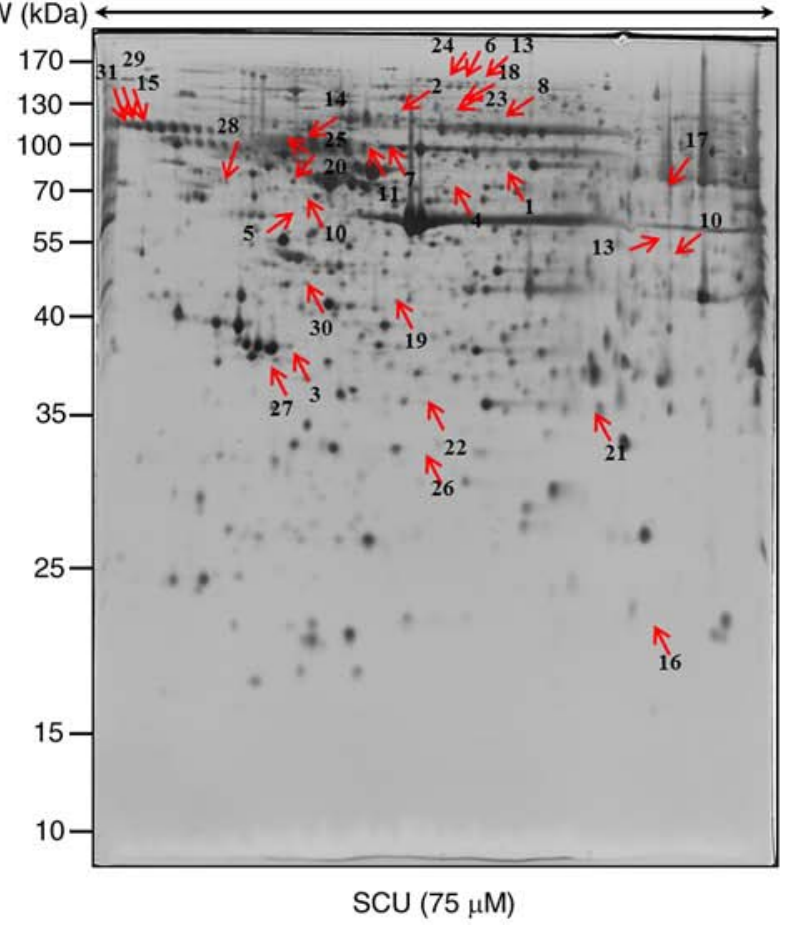

Figure 3. 2-DE protein pattern of differentially expressed proteins identified by MALDI-TOF-MS analysis in SNU484 cells. (A) Control (DMSO) and (B) SCU $(75 \mu \mathrm{M}) \mathrm{GC}$ SNU484 cells. The cells were treated with the indicated concentrations of SCU or DMSO for $24 \mathrm{~h}$. A total of $400 \mu \mathrm{g}$ of total proteins was separated on 18-cm linear IPG strips ( $\mathrm{pH} 4-7)$ by IEF and 12\% SDS-PAGE gels were used in the second dimension separation followed by silver staining of the gels. The arrows indicated by numbers are the protein spots identified successfully by MALDI-TOF-MS on protein database search. The experiments were performed in triplicate. 2-DE, two-dimensional gel electrophoresis; MALDI-TOF, matrix-assisted laser desorption/ ionization-time of flight; IEF, isoelectric focusing; GC, gastric cancer; SCU, Scutellarein; MW, molecular weight. 


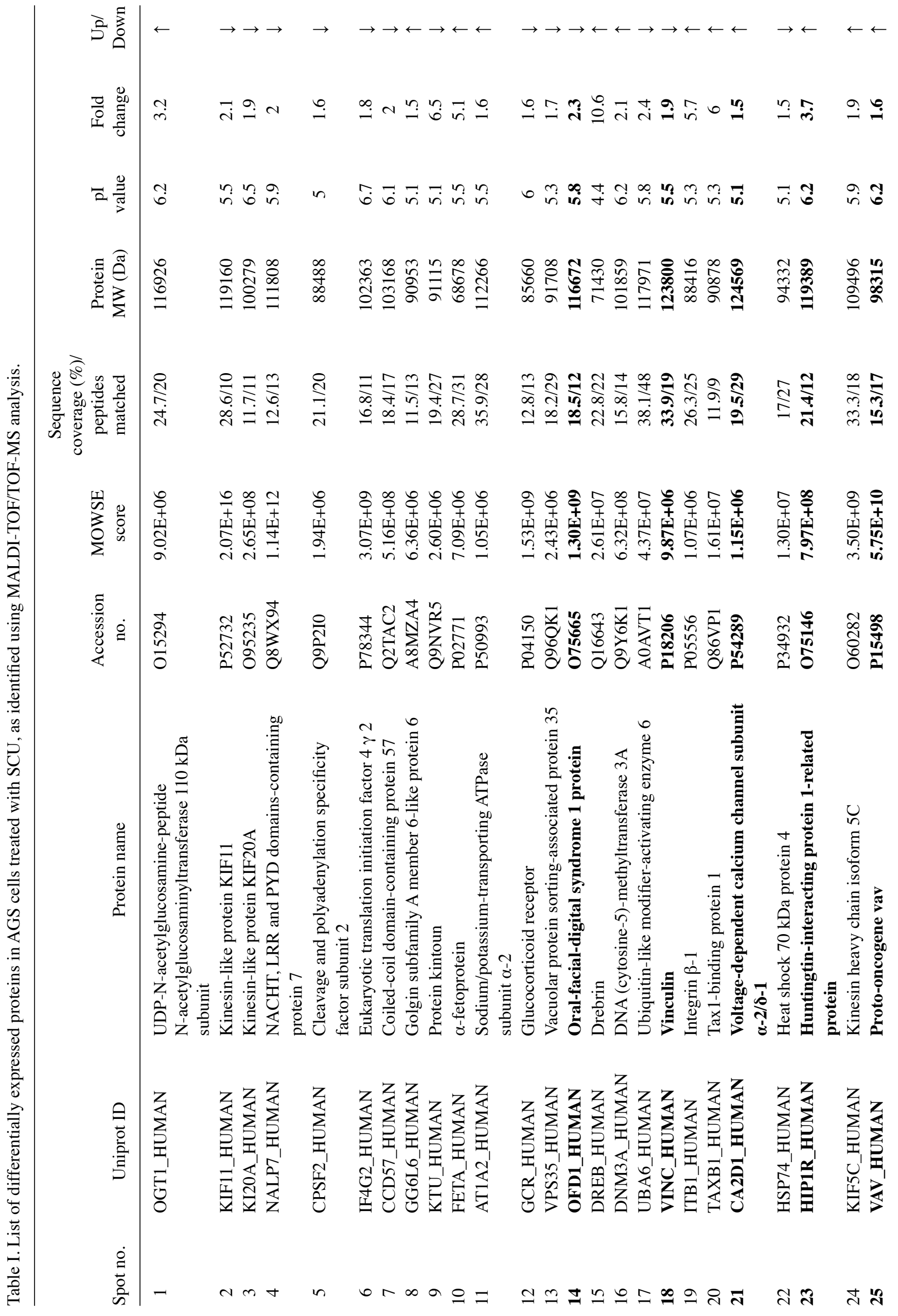




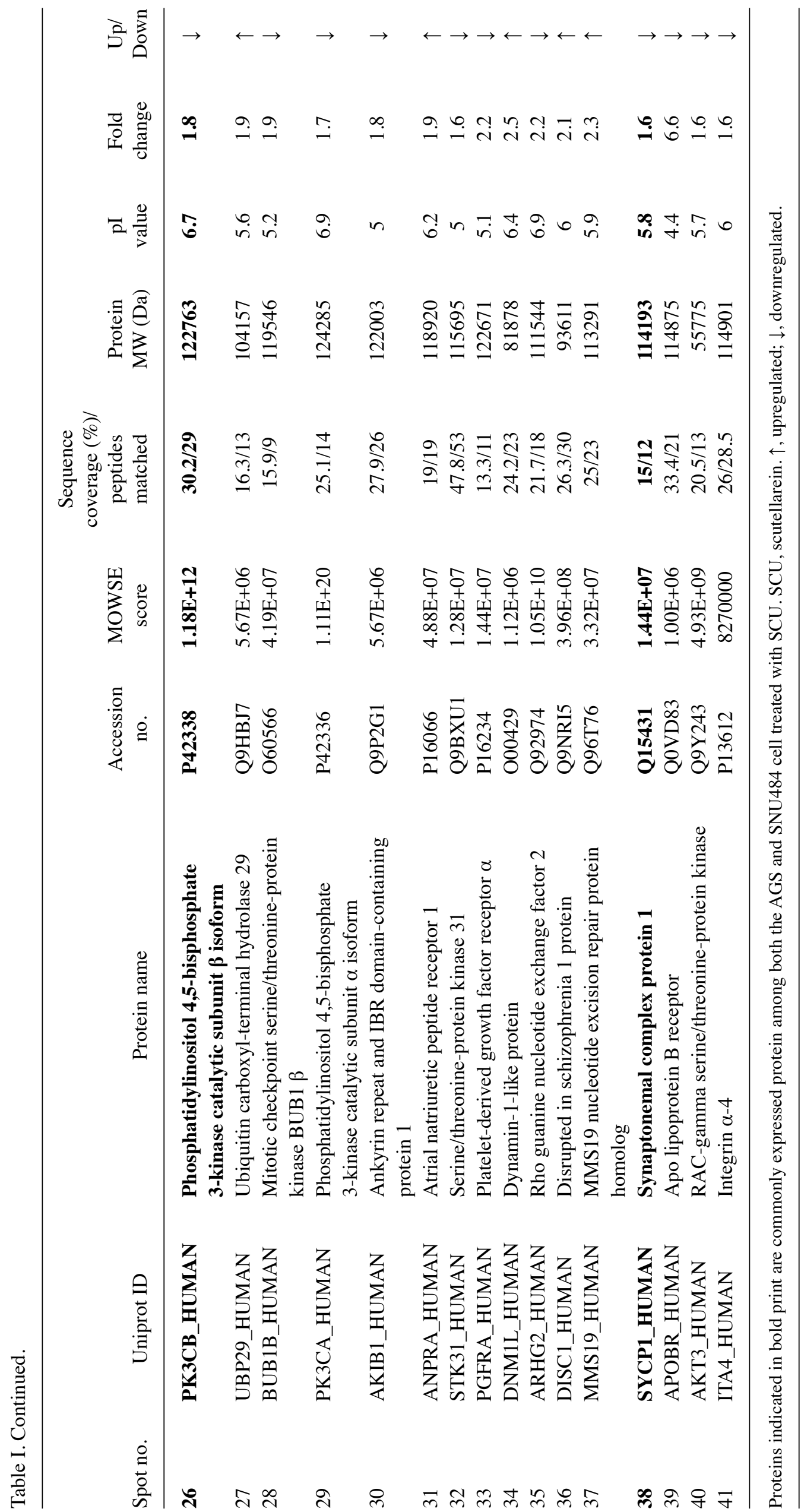




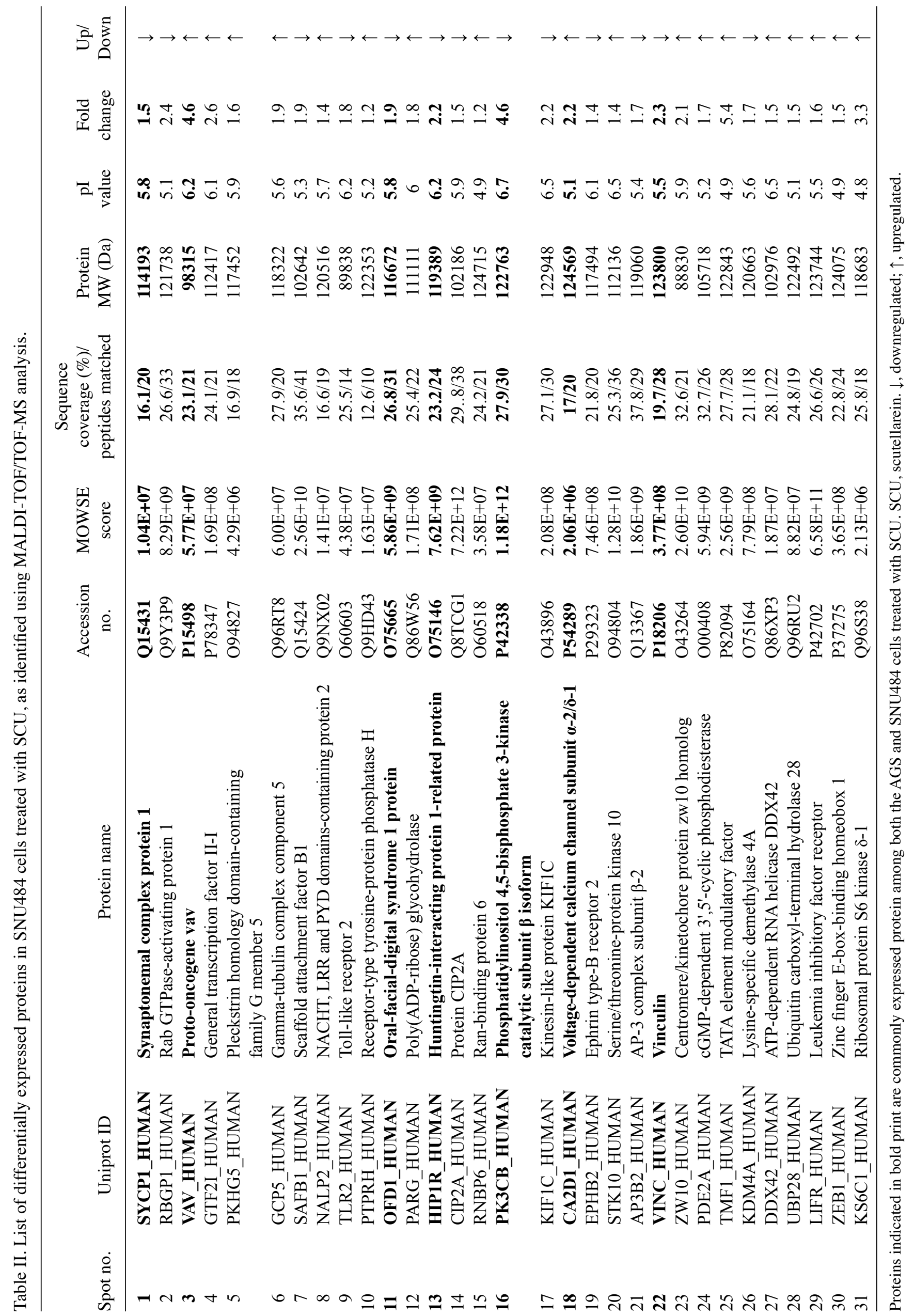


Table III. Number of interacting amino acid residues with the different Glide parameters of selected macromolecules with Scutellarein.

\begin{tabular}{lllccc}
\hline Sr. No. & Macromolecule & \multicolumn{1}{c}{ Interacting residues } & No of H-bonds & Glide G-Score & Glide energy \\
\hline 1 & CIP2A & Glu34; Val35; Gln82; AspB6 & 5 & -2.952 & -29.625 \\
2 & PIK3CB & Glu692; Ile685; Gln683; Lys636; Ser614 & 5 & -8.767 & -58.531 \\
3 & VINC & Lys236; Glu240; Lys708; Arg246; Glu243; & 5 & -6.049 & -54.679 \\
& & Ser656; Lys261; Lys666 & & & \\
4 & HIPR1 & ThrA:965; GluI:818; ArgI:963; SerF:955 & 4 & -4.678 & -47.516 \\
5 & VAV & Tyr56; Ser129; Thr75 & 6 & -6.666 & -59.988 \\
\hline
\end{tabular}

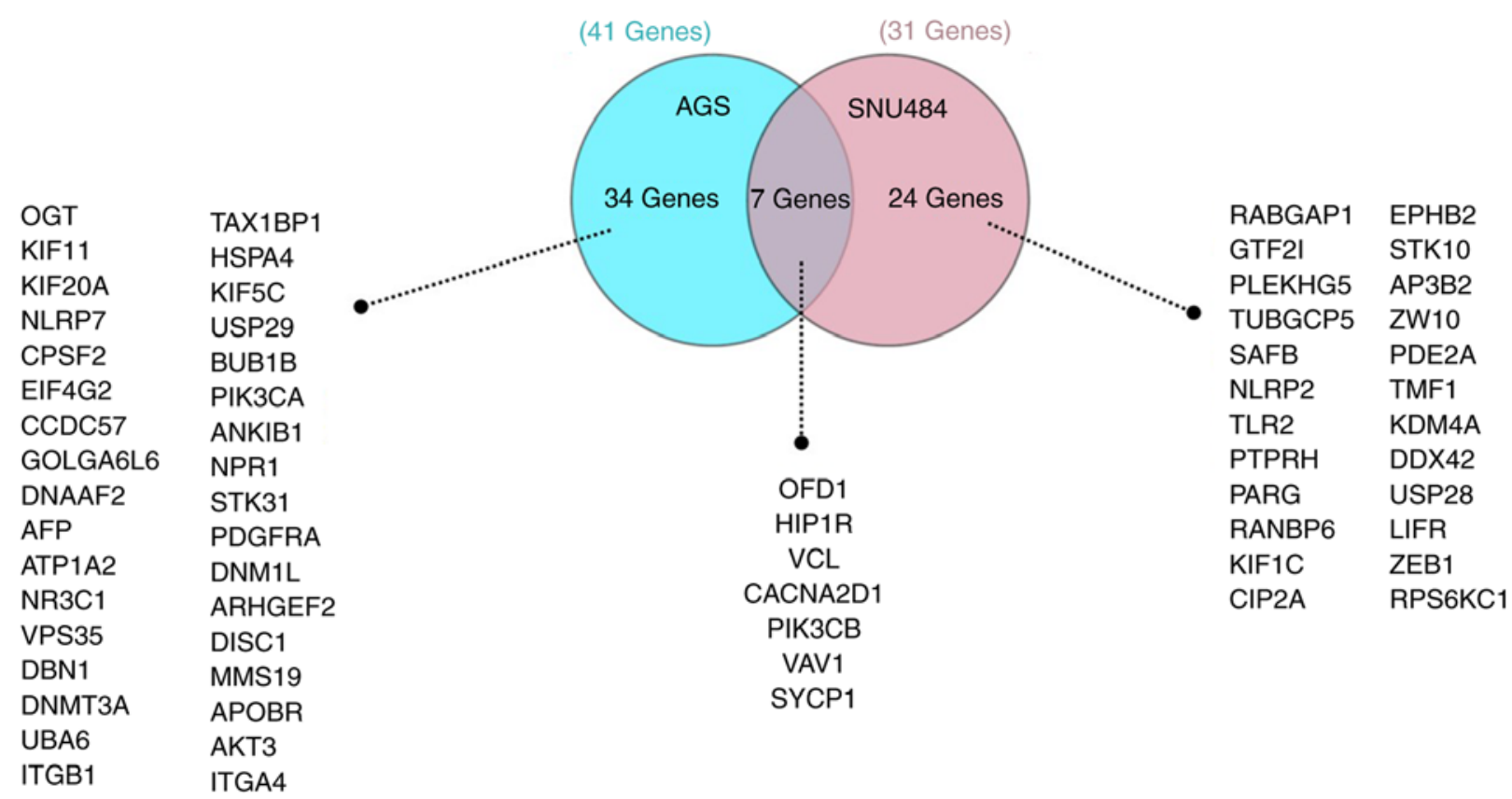

Figure 4. The differentially expressed proteins that overlapped between AGS and SNU484 cells are represented by a Venn diagram. All of the differentially expressed proteins from both GC cell lines treated with SCU were subjected to a comparative protein analysis for commonly expressed proteins using GENECODIS (http://genecodis.cnb.csic.es). Venn diagram of the number of differentially expressed proteins in both cell lines and commonly identified proteins upon treatment with SCU were identified. Implementation of comparative proteomics analysis yielded 7 [oral-facial-digital syndrome 1 protein (OFD1), vinculin (VINC), voltage-dependent calcium channel subunit $\alpha-2 / \delta-1$ (CACNA2D1), Huntingtin-interacting protein 1-related protein (HIP1R), proto-oncogene vav 1 (VAV1), phosphatidylinositol 4,5-bisphosphate 3-kinase catalytic subunit $\beta$ isoform (PIK3CB) and synaptonemal complex protein 1 (SYCP1)] commonly expressed proteins among the differentially expressed proteins between AGS and SNU484 cells treated with SCU. GC, gastric cancer; SCU, Scutellarein.

that the OFD1 protein cluster contains biological processes: Regulation of the G2/M transition of the mitotic cell cycle (GO:0010389), and cell differentiation (GO:0030154). VINC protein cluster contains biological processes: Negative regulation of apoptotic process (GO:0043066), regulation of cellular process (GO:0050794), positive regulation of cellular metabolic process (GO:0031325), and positive regulation of phosphatidylinositol 3-kinase activity (GO:0043552). CA2D1 protein cluster contains biological processes: Positive regulation of high voltage-gated calcium channel activity (GO:1901843), regulation of protein transport (GO:0051223), and homeostatic process (GO:0042592). HIP1R protein cluster contains biological processes: Negative regulation of apoptotic process (GO:0043066), negative regulation of cellular process (GO:0048523), and regulation of cytoskeleton organization (GO:0051493). PIK3CB protein cluster contains biological processes: Negative regulation of apoptotic process (GO:0043066), positive regulation of cellular metabolic process (GO:0031325), regulation of DNA-binding transcription factor activity (GO:0051090), and regulation of protein ubiquitination (GO:0031396). VAV1 protein cluster contains biological processes: Immune response-activating cell surface receptor signaling (GO:0002429), integrin-mediated signaling pathway (GO:0007229), and regulation of cell adhesion (GO:0030155). In addition to these proteins, we were interested in one more protein, which was found to be expressed in our previous studies and is related to proteomic analysis in GC cells treated with flavonoids (17). We found that protein CIP2A (CIP2A) was downregulated in SCU-treated SNU484 cells compared to the untreated group of cells. Protein cluster of CIP2A contains biological processes: Regulation of DNA metabolic process (GO:0051052), regulation of apoptotic 
Table IV. List of differentially expressed proteins involved in different biological processes in AGS cells treated with Scutellarein.

AGS cell line

\begin{tabular}{|c|c|c|c|}
\hline Sr. No. & Protein & Description & Biological process \\
\hline 1 & $\begin{array}{l}\text { VCL } \\
\text { VAV1 } \\
\text { PIK3CB } \\
\text { PIK3CA } \\
\text { PDGFRAV }\end{array}$ & $\begin{array}{l}\text { Vinculin } \\
\text { av guanine nucleotide exchange factor } 1 \\
\text { Phosphatidylinositol-4,5-bisphosphate 3-kinase catalytic subunit } \beta \\
\text { Phosphatidylinositol-4,5-bisphosphate 3-kinase catalytic subunit } \alpha \\
\text { Platelet derived growth factor receptor } \alpha\end{array}$ & $\begin{array}{l}\text { GO:0030168: platelet } \\
\text { activation }\end{array}$ \\
\hline 2 & $\begin{array}{l}\text { ITGB1 } \\
\text { VAV1 } \\
\text { PIK3CB } \\
\text { PIK3CA } \\
\text { PDGFRA }\end{array}$ & $\begin{array}{l}\text { Integrin subunit } \beta 1 \\
\text { Vav guanine nucleotide exchange factor } 1 \\
\text { Phosphatidylinositol-4,5-bisphosphate } 3 \text {-kinase catalytic subunit } \beta \\
\text { Phosphatidylinositol-4,5-bisphosphate 3-kinase catalytic subunit } \alpha \\
\text { Platelet derived growth factor receptor } \alpha\end{array}$ & $\begin{array}{l}\text { GO:0051897: positive } \\
\text { regulation of protein } \\
\text { kinase B signaling }\end{array}$ \\
\hline 3 & $\begin{array}{l}\text { DBN1 } \\
\text { VCL } \\
\text { ITGB1 } \\
\text { KIF5C } \\
\text { PIK3CB } \\
\text { PIK3CA } \\
\text { DNM1L } \\
\text { ITGA4 }\end{array}$ & $\begin{array}{l}\text { Drebrin } 1 \\
\text { Vinculin } \\
\text { Integrin subunit } \beta 1 \\
\text { Kinesin family member } 5 \mathrm{C} \\
\text { Phosphatidylinositol-4,5-bisphosphate } 3 \text {-kinase catalytic subunit } \beta \\
\text { Phosphatidylinositol-4,5-bisphosphate } 3 \text {-kinase catalytic subunit } \alpha \\
\text { Dynamin } 1 \text { like } \\
\text { Integrin subunit } \alpha 4\end{array}$ & $\begin{array}{l}\text { GO:0048667: cell } \\
\text { morphogenesis involved } \\
\text { in neuron differentiation }\end{array}$ \\
\hline 4 & $\begin{array}{l}\text { ATP1A2 } \\
\text { ITGB1 } \\
\text { CACNA2D1 } \\
\text { HIP1R } \\
\text { VAV1 } \\
\text { PIK3CB } \\
\text { PIK3CA } \\
\text { DNM1L } \\
\text { APOBR } \\
\text { ITGA4 }\end{array}$ & $\begin{array}{l}\text { ATPase } \mathrm{Na}+\mathrm{K}+\text { transporting subunit } \alpha 2 \\
\text { Integrin subunit } \beta 1 \\
\text { Calcium voltage-gated channel auxiliary subunit } \alpha 2 \delta 1 \\
\text { Huntingtin interacting protein } 1 \text { related } \\
\text { Vav guanine nucleotide exchange factor } 1 \\
\text { Phosphatidylinositol-4,5-bisphosphate } 3 \text {-kinase catalytic subunit } \beta \\
\text { Phosphatidylinositol-4,5-bisphosphate } 3 \text {-kinase catalytic subunit } \alpha \\
\text { Dynamin } 1 \text { like } \\
\text { Apolipoprotein B receptor } \\
\text { Integrin subunit } \alpha 4\end{array}$ & $\begin{array}{l}\text { GO:0098657: import } \\
\text { into cell }\end{array}$ \\
\hline 5 & $\begin{array}{l}\text { DNAAF2 } \\
\text { VPS35 } \\
\text { OFD1 } \\
\text { DBN1 } \\
\text { UBA6 } \\
\text { VCL } \\
\text { ITGB1 } \\
\text { KIF5C } \\
\text { PIK3CB } \\
\text { PIK3CA } \\
\text { DNM1L } \\
\text { DISC1 } \\
\text { ITGA4 }\end{array}$ & $\begin{array}{l}\text { Dynein axonemal assembly factor } 2 \\
\text { VPS35, retromer complex component } \\
\text { OFD1, centriole and centriolar satellite protein } \\
\text { Drebrin } 1 \\
\text { Ubiquitin like modifier activating enzyme } 6 \\
\text { Vinculin } \\
\text { Integrin subunit } \beta 1 \\
\text { Kinesin family member 5C } \\
\text { Phosphatidylinositol-4,5-bisphosphate 3-kinase catalytic subunit } \beta \\
\text { Phosphatidylinositol-4,5-bisphosphate 3-kinase catalytic subunit } \alpha \\
\text { Dynamin } 1 \text { like } \\
\text { DISC1 scaffold protein } \\
\text { Integrin subunit } \alpha 4\end{array}$ & $\begin{array}{l}\text { GO:0120036: plasma } \\
\text { membrane bounded cell } \\
\text { projection organization }\end{array}$ \\
\hline 6 & $\begin{array}{l}\text { KIF11 } \\
\text { KIF20A } \\
\text { DNAAF2 } \\
\text { ATP1A2 } \\
\text { OFD1 } \\
\text { VCL } \\
\text { ITGB1 } \\
\text { CACNA2D1 } \\
\text { KIF5C }\end{array}$ & $\begin{array}{l}\text { Kinesin family member } 11 \\
\text { Kinesin family member } 20 \mathrm{~A} \\
\text { Dynein axonemal assembly factor } 2 \\
\text { ATPase } \mathrm{Na}+\mathrm{K}+\text { transporting subunit } \alpha 2 \\
\text { OFD1, centriole and centriolar satellite protein } \\
\text { Vinculin } \\
\text { Integrin subunit } \beta 1 \\
\text { Calcium voltage-gated channel auxiliary subunit } \alpha 2 \delta 1 \\
\text { kinesin family member } 5 \mathrm{C}\end{array}$ & $\begin{array}{l}\text { GO:0006928: movement } \\
\text { of cell or subcellular } \\
\text { component }\end{array}$ \\
\hline
\end{tabular}


Table IV. Continued.

AGS cell line

\begin{tabular}{|c|c|c|c|}
\hline Sr. No. & Protein & Description & Biological process \\
\hline \multirow{21}{*}{7} & VAV1 & Vav guanine nucleotide exchange factor 1 & \multirow{21}{*}{$\begin{array}{l}\text { GO:0030030: cell } \\
\text { projection organization }\end{array}$} \\
\hline & PIK3CB & Phosphatidylinositol-4,5-bisphosphate 3-kinase catalytic subunit $\beta$ & \\
\hline & PIK3CA & Phosphatidylinositol-4,5-bisphosphate 3-kinase catalytic subunit $\alpha$ & \\
\hline & PDGFRA & Platelet derived growth factor receptor $\alpha$ & \\
\hline & ARHGEF2 & Rho/Rac guanine nucleotide exchange factor 2 & \\
\hline & DISC1 & DISC1 scaffold protein & \\
\hline & AKT3 & AKT serine/threonine kinase 3 & \\
\hline & ITGA4 & Integrin subunit $\alpha 4$ & \\
\hline & DNAAF2 & Dynein axonemal assembly factor 2 & \\
\hline & VPS35 & VPS35, retromer complex component & \\
\hline & OFD1 & OFD1, centriole and centriolar satellite protein & \\
\hline & DBN1 & Drebrin 1 & \\
\hline & UBA6 & Ubiquitin like modifier activating enzyme 6 & \\
\hline & $\mathrm{VCL}$ & Vinculin & \\
\hline & ITGB1 & Integrin subunit $\beta 1$ & \\
\hline & KIF5C & Kinesin family member $5 \mathrm{C}$ & \\
\hline & PIK3CB & Phosphatidylinositol-4,5-bisphosphate 3-kinase catalytic subunit $\beta$ & \\
\hline & PIK3CA & Phosphatidylinositol-4,5-bisphosphate 3-kinase catalytic subunit $\alpha$ & \\
\hline & DNM1L & Dynamin 1 like & \\
\hline & DISC1 & DISC1 scaffold protein & \\
\hline & ITGA4 & Integrin subunit $\alpha 4$ & \\
\hline \multirow[t]{12}{*}{8} & DBN1 & Drebrin 1 & \multirow{26}{*}{$\begin{array}{l}\text { GO:0048699: generation } \\
\text { of neurons }\end{array}$} \\
\hline & DNMT3A & DNA methyltransferase $3 \alpha$ & \\
\hline & UBA6 & Ubiquitin like modifier activating enzyme 6 & \\
\hline & VCL & Vinculin & \\
\hline & ITGB1 & Integrin subunit $\beta 1$ & \\
\hline & KIF5C & Kinesin family member $5 \mathrm{C}$ & \\
\hline & PIK3CB & Phosphatidylinositol-4,5-bisphosphate 3-kinase catalytic subunit $\beta$ & \\
\hline & PIK3CA & Phosphatidylinositol-4,5-bisphosphate 3-kinase catalytic subunit $\alpha$ & \\
\hline & DNM1L & Dynamin 1 like & \\
\hline & ARHGEF2 & Rho/Rac guanine nucleotide exchange factor 2 & \\
\hline & DISC1 & DISC1 scaffold protein & \\
\hline & ITGA4 & Integrin subunit $\alpha 4$ & \\
\hline \multirow[t]{14}{*}{9} & DNAAF2 & Dynein axonemal assembly factor 2 & \\
\hline & ATP1A2 & ATPase $\mathrm{Na}+/ \mathrm{K}+$ transporting subunit $\alpha 2$ & \\
\hline & VPS35 & VPS35, retromer complex component & \\
\hline & VCL & Vinculin & \\
\hline & ITGB1 & Integrin subunit $\beta 1$ & \\
\hline & KIF5C & Kinesin family member $5 \mathrm{C}$ & \\
\hline & VAV1 & Vav guanine nucleotide exchange factor 1 & \\
\hline & PIK3CB & Phosphatidylinositol-4,5-bisphosphate 3-kinase catalytic subunit $\beta$ & \\
\hline & PIK3CA & Phosphatidylinositol-4,5-bisphosphate 3-kinase catalytic subunit $\alpha$ & \\
\hline & PDGFRA & Platelet derived growth factor receptor $\alpha$ & \\
\hline & ARHGEF2 & Rho/Rac guanine nucleotide exchange factor 2 & \\
\hline & DISC1 & DISC1 scaffold protein & \\
\hline & AKT3 & AKT serine/threonine kinase 3 & \\
\hline & ITGA4 & Integrin subunit $\alpha 4$ & \\
\hline \multirow[t]{3}{*}{10} & OGT & O-linked $\mathrm{N}$-acetylglucosamine (GlcNAc) transferase & \multirow{3}{*}{$\begin{array}{l}\text { GO:0006915: apoptotic } \\
\text { process }\end{array}$} \\
\hline & $\mathrm{NR} 3 \mathrm{C} 1$ & Nuclear receptor subfamily 3 group C member 1 & \\
\hline & VPS35 & VPS35, retromer complex component & \\
\hline
\end{tabular}


Table IV. Continued.

AGS cell line

\begin{tabular}{|c|c|c|c|}
\hline Sr. No. & Protein & Description & Biological process \\
\hline & DNMT3A & DNA methyltransferase $3 \alpha$ & \\
\hline & ITGB1 & Integrin subunit $\beta 1$ & \\
\hline & TAX1BP1 & Tax 1 binding protein 1 & \\
\hline & HIP1R & Huntingtin interacting protein 1 related & \\
\hline & VAV1 & Vav guanine nucleotide exchange factor 1 & \\
\hline & PIK3CB & Phosphatidylinositol-4,5-bisphosphate 3-kinase catalytic subunit $\beta$ & \\
\hline & BUB1B & BUB1 mitotic checkpoint serine/threonine kinase B & \\
\hline & PIK3CA & Phosphatidylinositol-4,5-bisphosphate 3-kinase catalytic subunit $\alpha$ & \\
\hline & PDGFRA & Platelet derived growth factor receptor $\alpha$ & \\
\hline & DNM1L & Dynamin 1 like & \\
\hline & ARHGEF2 & Rho/Rac guanine nucleotide exchange factor 2 & \\
\hline & ITGA4 & Integrin subunit $\alpha 4$ & \\
\hline
\end{tabular}

process (GO:0042981), negative regulation of stress-activated MAPK cascade (GO:0032873) and mitotic cell cycle process (GO:1903047).

Validation of selected proteins by immunoblotting analysis. To verify the identified proteins from 2-DE, the immunoblotting analysis was performed in the SCU-treated AGS and SNU484 cells for a few selected important proteins. Fig. 5 shows that the western blot analysis revealed significantly decreased protein expression of OFD1, VCL, PIK3CB, HIP1R, and CIP2A in both the SCU-treated GC cell lines, in comparison with the control groups $(\mathrm{P}<0.05)$. These data confirmed that the results of the immunoblotting were consistent with those of the 2-DE outcome. The comparative proteomic analysis and the confirmation of these proteins by western blot analysis increased the confidence in the obtained results from the 2-DE analysis to consider these proteins as biomarker candidates.

Molecular docking studies confirm the binding affinity of SCU with biomarker candidates. Molecular docking analysis against Scutellarein revealed that all the significant protein macromolecules chosen form a stable H-bond with Scutellarein. Table III shows the Glide parameters, such as Glide G-Score and Glide energy of the significant protein molecules with the structure of SCU along with the number of H-bonds, interacting with the amino acids. The Glide score describes the perfect fit for a ligand in the active site of the target molecule, and gives the efficiency of the molecular binding. The Glide score of the target molecules, PIK3CB, HIPR1, CIP2A, VAV1 and VINC, were found to be $-8.767,-4.678,-2.952,-6.666$, and -6.049 ), respectively. Fig. 6 shows the molecular binding models of the three significant target molecules, PIK3CB, HIPR1 and VINC, with their ligand interaction diagram. The LigPlot shows the binding with their appropriate interacting amino acids with stable Vander Waals force and H-bonding. PIK3CB interacted with SCU by forming five stable hydrogen bonds with amino acids: Glu692, Ile685, Gln683, Lys636, and Ser614. Vinculin formed interactions with the following residues: Lys236,
Glu240, Lys708, Arg246, Glu243, Ser656, Lys261, and Lys666; and HIP1R interacts with ThrA965, GluI818, ArgI963, and SerF:955. With consideration of the Glide parameters of all the target molecules with SCU, the interaction of PIK3CB has shown more significant binding with a Glide score of -8.767 forming stable affinity, and is considered as the best fit among our proteins in the study. Together with this, Fig. S2 shows the molecular binding models of the proteins CIP2A and VAV with their ligand interaction with SCU. CIP2A shows interaction with SCU by forming five hydrogen bonds, interacting with amino acids: Glu34, Val35, Gln82, and AspB6; whereas, protein VAV showed interaction with only a few amino acids: Tyr56, Ser129, and Thr75 compared with the other structures.

Functional classifications of the identified differentially expressed proteins. The Gene Ontology bioinformatics tool was useful to facilitate the interpretation of the proteomics data. WebGestalt (http://www.webgestalt.org ) tool was used to analyze the differentially identified proteins from both cell lines treated with SCU in terms of biological process, cellular component and molecular function by GOSlim view. Fig. 7A (AGS) and Fig. 7B (SNU484) show the distribution of the number of proteins to several categories of the functional annotation. Among the GO categories, biological processes enrichment of these differentially protein from both cell lines were viewed independently with WebGestalt with the condition of P-value $<0.05$. Table IV (AGS) and Table V (SNU) show that a total of 10 biological processes were significant in both the cell lines treated with SCU. Apoptotic process (GO:0006915) was found to be the leading biological process in both cell lines treated with SCU, with 15 proteins in AGS cells and 11 proteins in SNU484 cells being involved. GO analysis showed that $\mathrm{SCU}$ could affect proteins that regulate a broad range of molecular functions, such as protein binding and ion binding. We uploaded 41 (AGS) and 31 (SNU484) differentially expressed proteins on to the PANTHER database for pathway enrichment, which identified 25 and 24 pathways with signaling mechanisms that are concerned with 
Table V. List of differentially expressed proteins involved in different biological processes in SNU484 cells treated with Scutellarein.

SNU484 cell line

\begin{tabular}{|c|c|c|c|}
\hline Sr. No. & Protein & Description & Biological process \\
\hline 1 & $\begin{array}{l}\text { TLR2 } \\
\text { PDE2A }\end{array}$ & $\begin{array}{l}\text { Toll like receptor } 2 \\
\text { Phosphodiesterase } 2 \mathrm{~A}\end{array}$ & $\begin{array}{l}\text { GO:0036005: response to } \\
\text { macrophage colony- } \\
\text { stimulating factor }\end{array}$ \\
\hline 2 & $\begin{array}{l}\text { TLR2 } \\
\text { PDE2A }\end{array}$ & $\begin{array}{l}\text { Toll like receptor } 2 \\
\text { Phosphodiesterase } 2 \mathrm{~A}\end{array}$ & $\begin{array}{l}\text { GO:0036006: cellular response to } \\
\text { macrophage colony-stimulating } \\
\text { factor stimulus }\end{array}$ \\
\hline 3 & $\begin{array}{l}\text { HIP1R } \\
\text { PIK3CB }\end{array}$ & $\begin{array}{l}\text { Huntingtin interacting protein } 1 \text { related } \\
\text { Phosphatidylinositol-4,5-bisphosphate 3-kinase } \\
\text { catalytic subunit } \beta\end{array}$ & $\begin{array}{l}\text { GO:2000369: regulation of } \\
\text { clathrin-dependent endocytosis }\end{array}$ \\
\hline 4 & $\begin{array}{l}\text { SYCP1 } \\
\text { TMF1 }\end{array}$ & $\begin{array}{l}\text { Synaptonemal complex protein } 1 \\
\text { TATA element modulatory factor } 1\end{array}$ & $\begin{array}{l}\text { GO:0007289: spermatid nucleus } \\
\text { differentiation }\end{array}$ \\
\hline 5 & $\begin{array}{l}\text { TUBGCP5 } \\
\text { OFD1 } \\
\text { PIBF1 }\end{array}$ & $\begin{array}{l}\text { Tubulin gamma complex associated protein } 5 \\
\text { OFD1, centriole and centriolar satellite protein } \\
\text { Progesterone immunomodulatory binding factor } 1\end{array}$ & $\begin{array}{l}\text { GO:0090307: mitotic spindle } \\
\text { assembly }\end{array}$ \\
\hline 6 & $\begin{array}{l}\text { TUBGCP5 } \\
\text { OFD1 } \\
\text { ZW10 } \\
\text { PIBF1 }\end{array}$ & $\begin{array}{l}\text { Tubulin } \gamma \text { complex associated protein } 5 \\
\text { OFD1, centriole and centriolar satellite protein } \\
\text { zW } 10 \text { kinetochore protein } \\
\text { Progesterone immunomodulatory binding factor } 1\end{array}$ & $\begin{array}{l}\text { GO: } 1902850 \text { : microtubule } \\
\text { cytoskeleton organization involved } \\
\text { in mitosis }\end{array}$ \\
\hline 7 & $\begin{array}{l}\text { TUBGCP5 } \\
\text { OFD1 } \\
\text { PIBF1 }\end{array}$ & $\begin{array}{l}\text { Tubulin } \gamma \text { complex associated protein } 5 \\
\text { OFD1, centriole and centriolar satellite protein } \\
\text { Progesterone immunomodulatory binding factor } 1\end{array}$ & $\begin{array}{l}\text { GO:0007052: mitotic spindle } \\
\text { organization }\end{array}$ \\
\hline 8 & $\begin{array}{l}\text { TUBGCP5 } \\
\text { OFD1 } \\
\text { PIBF1 }\end{array}$ & $\begin{array}{l}\text { Tubulin } \gamma \text { complex associated protein } 5 \\
\text { OFD1, centriole and centriolar satellite protein } \\
\text { Progesterone immunomodulatory binding factor } 1\end{array}$ & GO:0051225: spindle assembly \\
\hline 9 & $\begin{array}{l}\text { SYCP1 } \\
\text { TUBGCP5 } \\
\text { OFD1 } \\
\text { ZW10 } \\
\text { PIBF1 }\end{array}$ & $\begin{array}{l}\text { Synaptonemal complex protein } 1 \\
\text { Tubulin } \gamma \text { complex associated protein } 5 \\
\text { OFD1, centriole and centriolar satellite protein } \\
\text { zw10 kinetochore protein } \\
\text { Progesterone immunomodulatory binding factor } 1\end{array}$ & GO:0000280: nuclear division \\
\hline 10 & $\begin{array}{l}\text { PLEKHG5 } \\
\text { NLRP2 } \\
\text { TLR2 } \\
\text { PTPRH } \\
\text { HIP1R } \\
\text { PIK3CB }\end{array}$ & $\begin{array}{l}\text { Pleckstrin homology and RhoGEF domain containing G5 } \\
\text { NLR family pyrin domain containing } 2 \\
\text { Toll like receptor } 2 \\
\text { Protein tyrosine phosphatase, receptor type H } \\
\text { Huntingtin interacting protein } 1 \text { related } \\
\text { Phosphatidylinositol-4,5-bisphosphate } 3 \text {-kinase catalytic } \\
\text { subunit } \beta \\
\text { EPH receptor B2 } \\
\text { TATA element modulatory factor } 1 \\
\text { DEAD-box helicase } 42 \\
\text { Ubiquitin specific peptidase } 28\end{array}$ & GO:0006915: apoptotic process \\
\hline
\end{tabular}

the effect of SCU on AGS and SNU484 cancer cells, respectively as shown in Figs. 8 (AGS) and 9 (SNU484). Along with the commonly differentiated proteins in both the cell lines AGS and SNU484, there are three additional proteins (AKT3, ITGB1, and PIK3CA) expressed only in AGS which is found to be vital in contributing to the cellular mechanisms of the enriched pathways. Tables SI and SII shows the detailed individual pathways with protein distribution in AGS and SNU484 cell lines.

\section{Discussion}

Recent developments in proteome comparative analysis are intermittently used in the identification of protein expression 

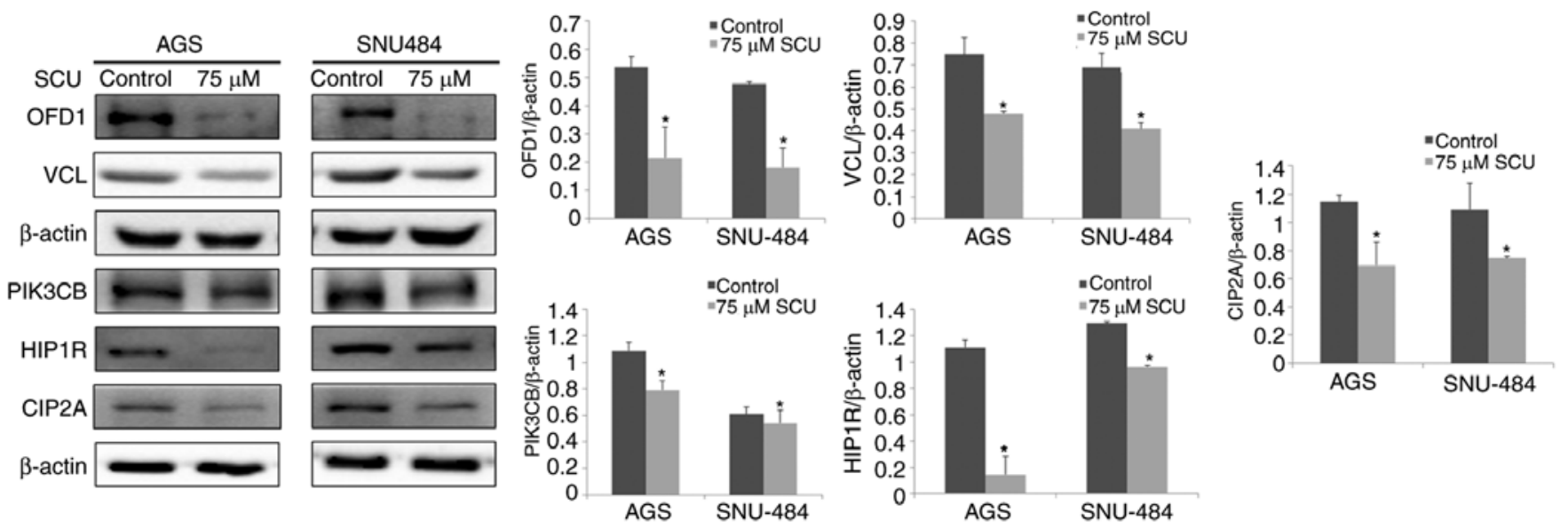

Figure 5. Western blot analysis confirmation of differentially expressed proteins. (A) AGS and (B) SNU484 GC cell lines were treated with control (DMSO) or SCU $(75 \mu \mathrm{M})$, incubated for $24 \mathrm{~h}$ and protein samples were prepared and separated on 10-12\% SDS-PAGE. OFD1, VCL, PIK3CB, HIP1R and CIP2A proteins were assessed using the respective antibodies. For the loading control $\beta$-actin was used and normalized to measure the expression changes. The bands are representative of three independent experiments [ ${ }^{*} \mathrm{P}<0.05$, significant difference vs. the control (DMSO)]. GC, gastric cancer; SCU, Scutellarein; OFD1, oral-facial-digital syndrome 1 protein; VCL, vinculin; PIK3CB, phosphatidylinositol 4,5-bisphosphate 3-kinase catalytic subunit $\beta$ isoform; HIP1R, Huntingtin-interacting protein 1-related protein; CIP2A, cancerous inhibitor of protein phosphatase $2 \mathrm{~A}$.

A

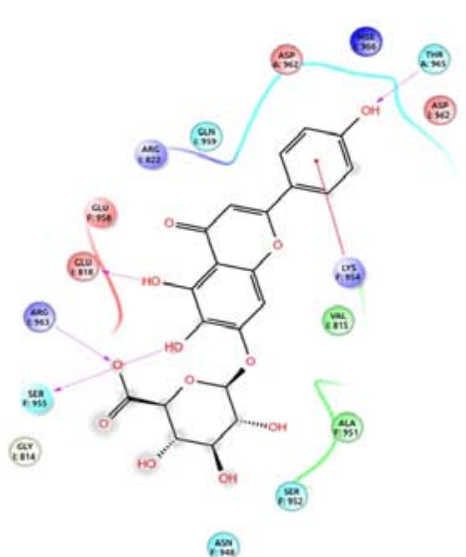

B

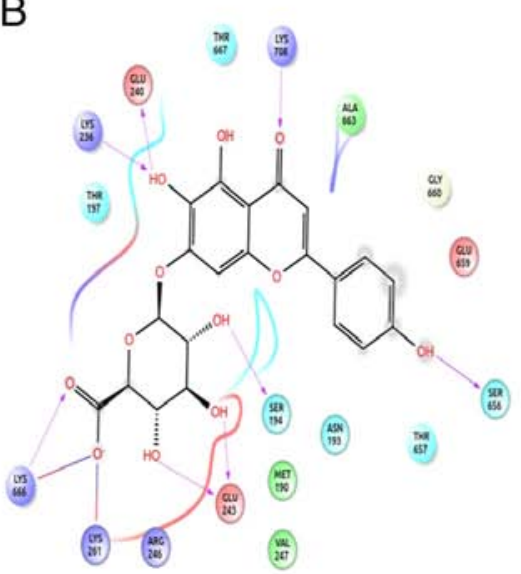

C
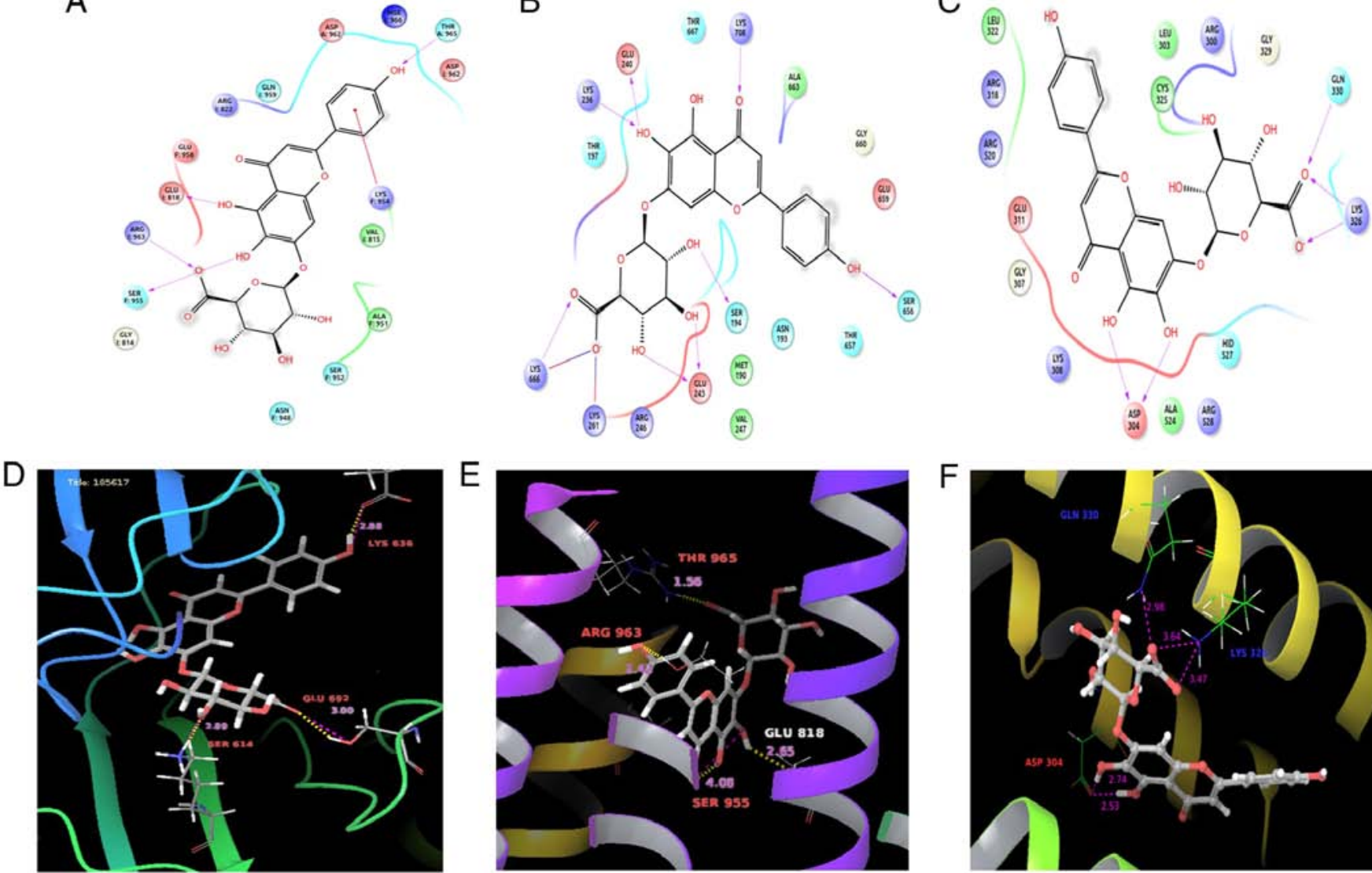

Figure 6. Ligand-interaction and Molecular docking of the target proteins with SCU. The amino acid residues in the proteins showing stable hydrogen bonding with SCU and molecular binding models of the structural complex. (A) The LigPlot of protein PIK3CB with SCU. (B) The LigPlot of protein HIP1R with SCU. (C) The LigPlot of protein VCL with SCU. (D) The binding model of protein PIK3CB complexed with SCU. (E) The binding model of protein HIP1R complexed with SCU. (F) The binding model of protein VCL complexed with SCU. SCU, Scutellarein; PIK3CB, phosphatidylinositol 4,5-bisphosphate 3-kinase catalytic subunit $\beta$ isoform; HIP1R, Huntingtin-interacting protein 1-related protein; VCL, vinculin.

alterations upon drug treatment of cancer cells (9). These strands of evidence could provide clues for the investigation of the effects of drug treatment and further provide better knowledge of the molecular mechanism of action of agents. In the current study, two gastric cancer (GC) cell lines were used as in vitro models; the data presented in our present and 
A

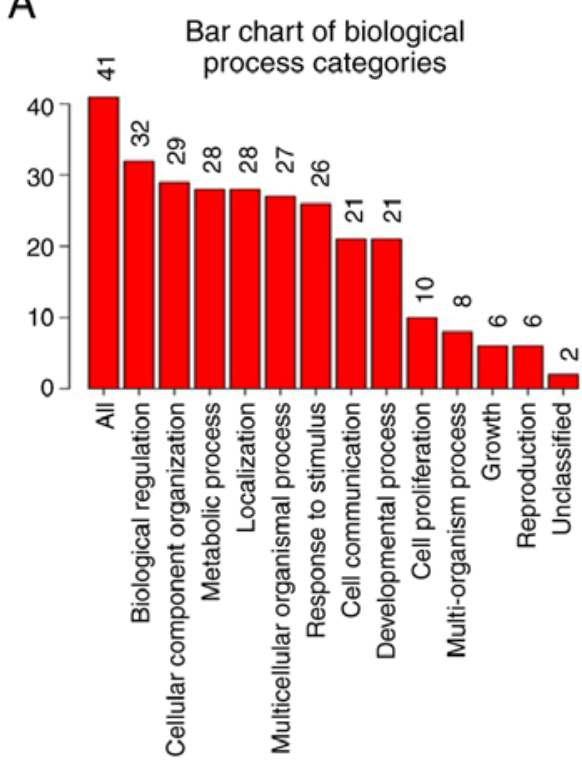

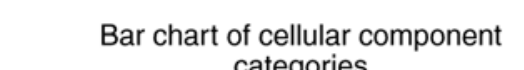

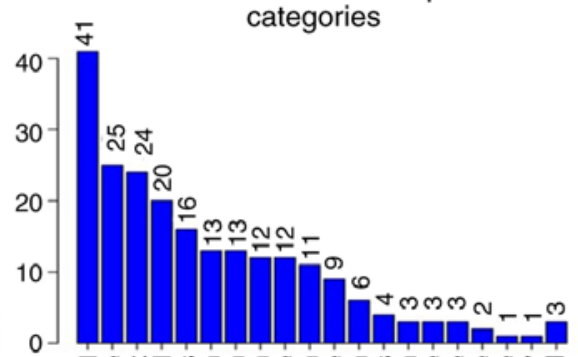

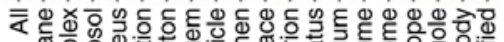

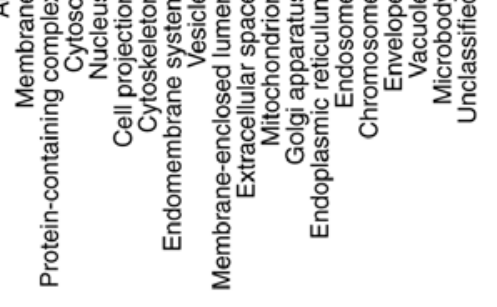

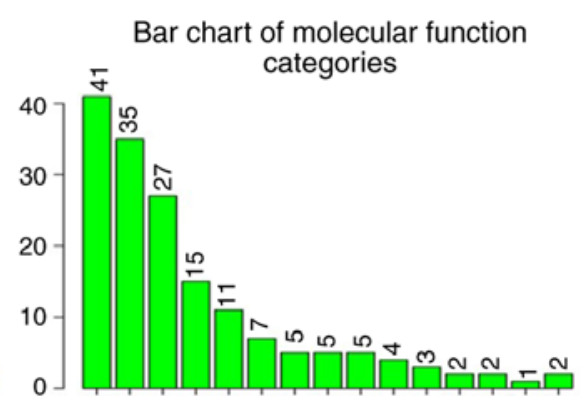

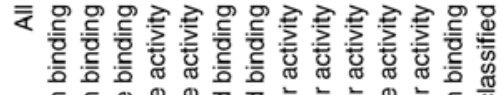

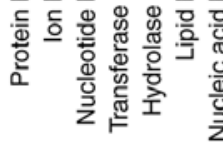

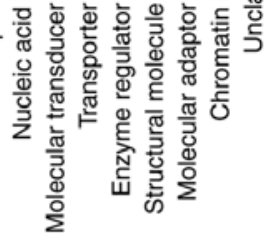

B

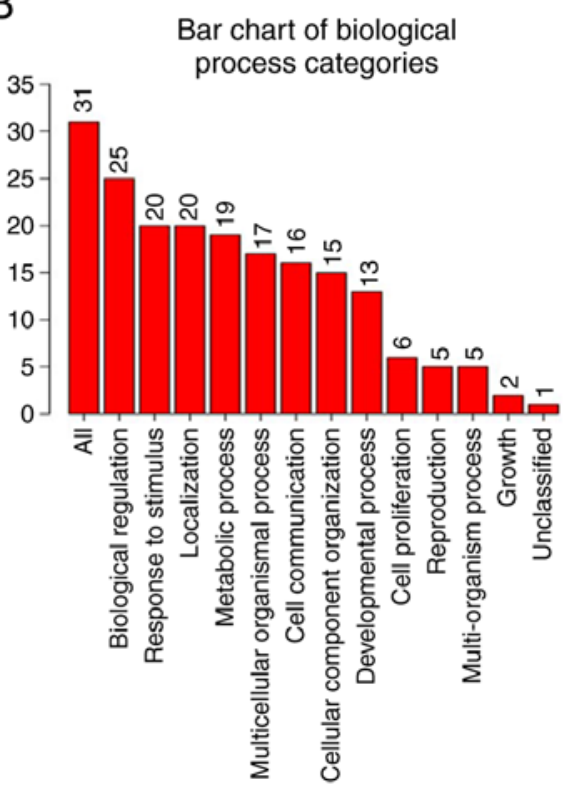

Bar chart of cellular component

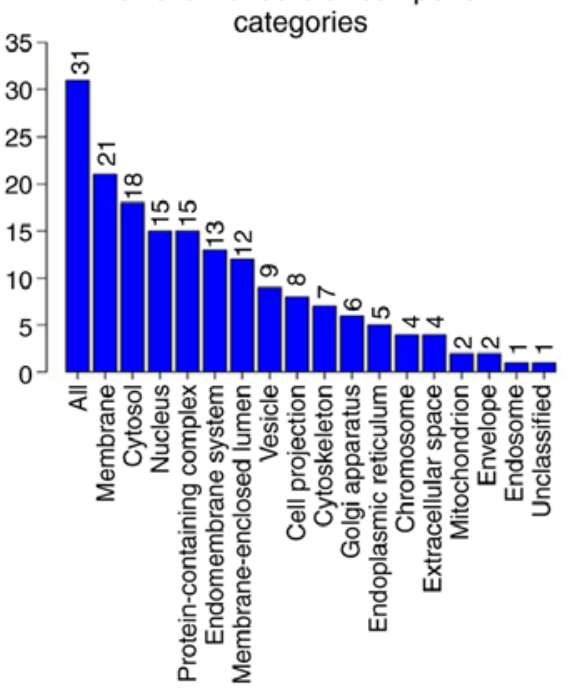

Bar chart of molecular function

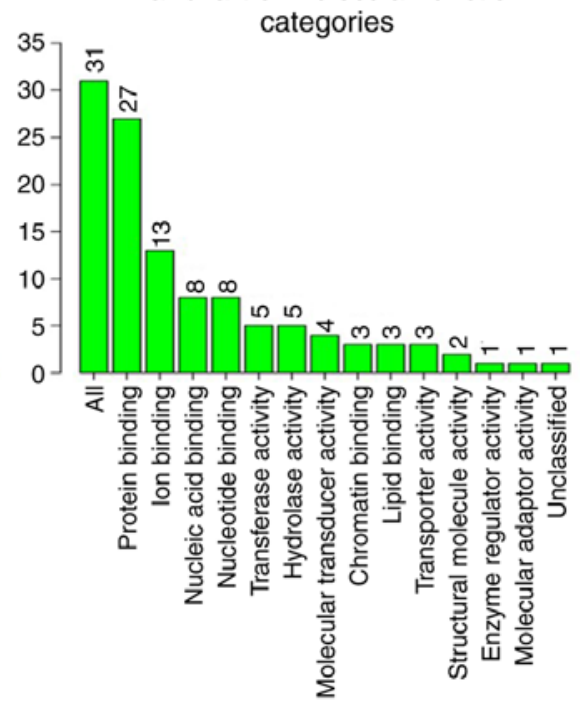

Figure 7. GOSlim summary of the proteins differentially altered in the GC cell lines (A) AGS and (B) SNU484 cells treated with SCU. The GO profile of differential expressed proteins in both the cell lines grouped in terms of Biological process, Cellular component and Molecular function by using WebGestalt (http://www.webgestalt.org).

previous studies showed that Scutellarein (SCU) significantly inhibited cell proliferation and induced apoptosis in both GC cell lines. A higher proportion of the proteome is expected to be involved in this comparative proteomic approach thereby increasing the opportunities for the discovery of protein biomarkers in GC cell response to SCU treatment. To analyze the alterations at the protein level, a comparative proteomic technique using 2-DE coupled with mass spectrometry was implemented to find the modified proteins in GC cells in response to SCU treatment. In AGS cells, a total of 41 differentially expressed proteins, and in SNU484, a total of 31 differentially expressed proteins were identified successfully by MALDI/TOF-MS analysis. All the proteins spots were not identified successfully because of the limitations and relatively low concentrations in mass spectrometry. Among the differentially expressed proteins, 5 critical proteins were confirmed by immunoblotting by commercially available antibodies, and 5 of these (PIK3CB, HIPR1, VINC, CIP2A and VAV) were studied for their binding affinity with SCU using molecular simulation. The Glide scores and energy values showed optimum values, and significantly confirmed the efficiency of the compound SCU to bind with the significantly expressed proteins in the present study. Of note, structurally, the presence of four hydroxyl groups in the compound SCU makes it more stable for binding and affinity to form hydrogen bonding interactions with the molecular targets (25). The identified proteins were found to be predominantly involved in the process of tumor growth, cell cycle regulation, and apoptosis in cancer cells. Implementation of comparative proteomics analysis yielded 7 [oral-facial-digital syndrome 1 protein (OFD1), vinculin (VINC), voltage-dependent calcium channel subunit $\alpha-2 / \delta-1$ (CACNA2D1), Huntingtin-interacting 


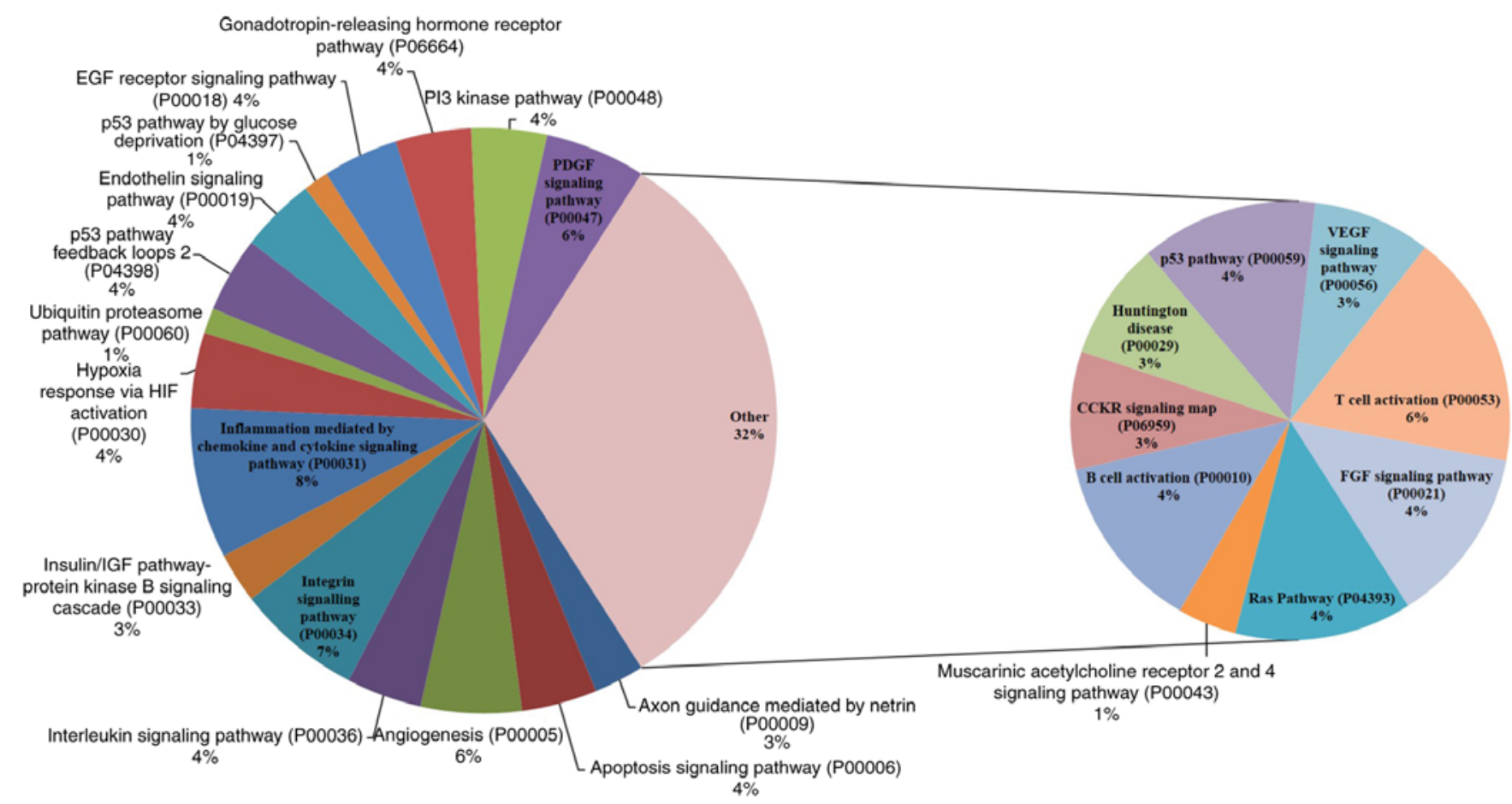

Figure 8. Pathway enrichment of the differentially altered proteins in GC AGS cells treated with SCU. The significantly altered proteins in AGS cells treated with SCU when compared with the untreated group of cells were grouped based on the enriched pathways using PANTHER database (http://pantherdb.org/). The percentage (\%) of proteins participating in each pathway are shown using a pie chart. GC, gastric cancer; SCU, Scutellarein.

protein 1-related protein (HIP1R), proto-oncogene vav 1 (VAV1), phosphatidylinositol 4,5-bisphosphate 3-kinase catalytic subunit $\beta$ isoform (PIK3CB) and synaptonemal complex protein 1 (SYCP1)] commonly expressed proteins among the differentially expressed proteins between AGS and SNU484 cells treated with SCU along with CIP2A (SNU484), which attracted our interest since dysregulation of its function and expression is correlated with cancer progression, tumorigenesis, and apoptosis $(26,27)$. To the best of our knowledge, the anticancer effects of SCU on expression of these genes have not previously been reported in GC cells. GO biological process and PANTHER pathway analysis revealed that all 7 proteins appear to participate in major biological processes such as apoptosis, cellulare movement or subcellular component, and also appear in several pathways stimulated by SCU in both cell lines.

Initially, OFD1 was found to be expressed in oral-facial-digital syndrome, and is subject to ciliopathies such as retinitis pigmentosa, and Simpson-Golabi-Behmel syndrome type 2 (28). OFD1 is essential for primary cilia formation, and depletion of OFD1 results in the loss of primary cilia. The contribution of primary cilium function to tumorigenesis is complex; studies recommend that dissecting the regulatory mechanisms of OFD1 will provide insight into the tumorigenesis functions and offer potential new therapeutic tools for the treatment of cancers. In our present results, OFD1 was downregulated in both GC cell lines treated with SCU, and functional analysis showed its involvement in mitotic spindle assembly, nuclear division, and microtubule cytoskeleton organization involved in mitosis. PIK3CB, an isoform of the catalytic subunit of phosphoinositide 3-kinase (PI3K), is expressed to be oncogenic in their wild-type form. The depletion of PIK3CB isoform encodes for p110, which was found to inactivate the PTEN pathway, followed by the inhibition of growth in both in vitro and in vivo environments in cancer models (29). PI3K $\beta$ activation is responsible for the alteration in PIK3CB expression, which may lead to an elevation in cancer survival and cellular proliferation. PI3K $\beta$ may be involved directly in cell invasion and migration through interactions with other proteins, clathrin and integrins, which play key roles in assisting cell motility function $(30,31)$. In the present study, the results revealed that SCU abated the PIK3CB protein expression in both GC cell lines, when compared to the control cell group. The decreased expression of PIK3CB may be explained by its binding affinity with SCU. GO analysis results showed the greater role of PIK3CB involvement in the maximum category of the biological process as well as being a prominent candidate of all the pathways identified by PANTHER analysis in both cell lines. From our previous study, GC cells treated with Pectolinarigenin, a natural flavonoid, also showed downregulation of PIK3CB protein expression accompanied by a distinct group of cellular functions, including cell growth and proliferation (17). HIP1R is known as an endocytic adaptor with its functional roles in vesicle trafficking and clathrin-mediated endocytosis. Studies have shown that the downregulation of HIP1R in aggressive prostate cancer cell lines recapped the anti-metastatic effects of miR-23b/miR-27b, including decreased migration and anchorage-independent growth (32). The present results indicate that SCU downregulated the expression of HIP1R protein in AGS and SNU484 cells compared with the untreated group of cells. GO analysis of differentially expressed proteins from both cell lines showed the involvement of HIP1R proteins in different biological processes such as import into the cell, 


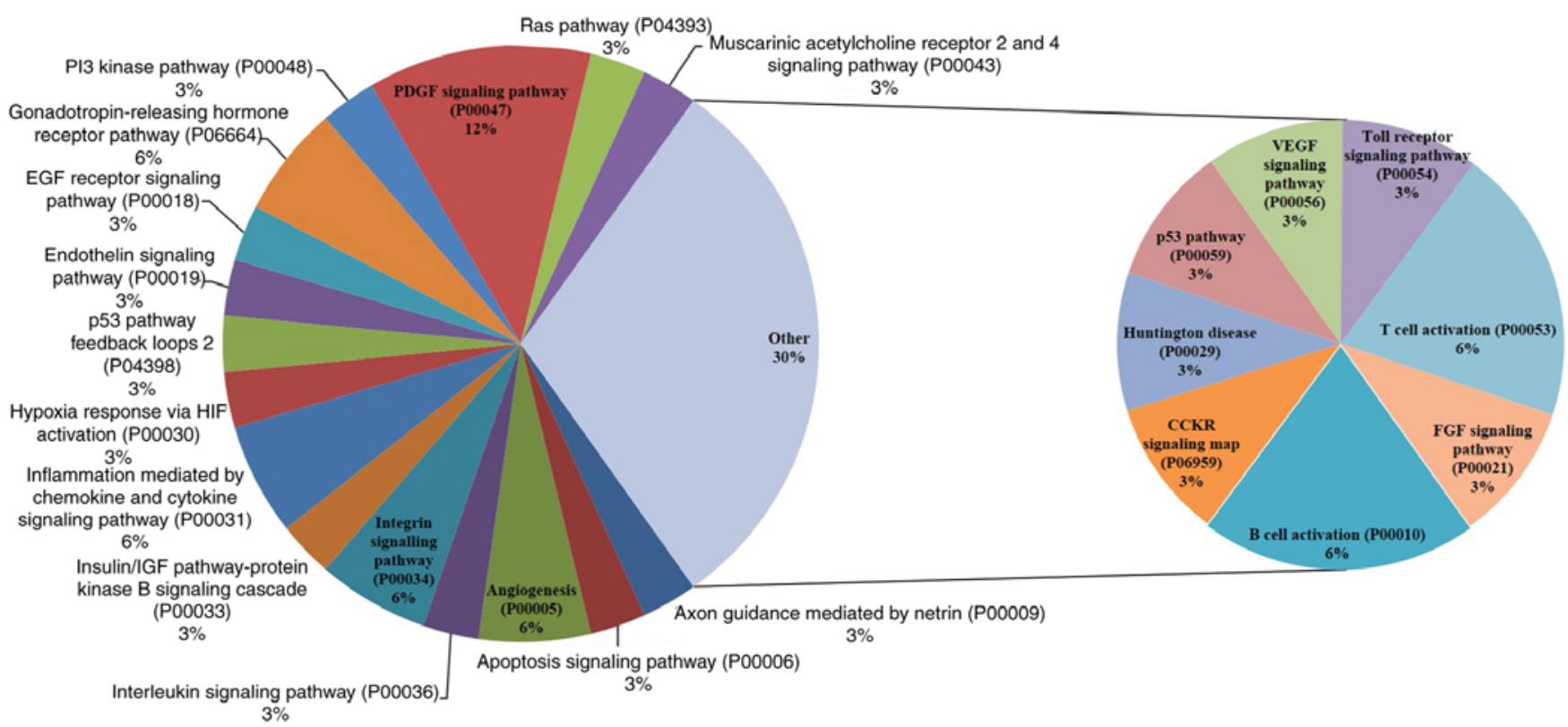

Figure 9. Pathway enrichment of the differentially altered proteins in GC SNU484 cells treated with SCU. The significantly altered proteins in SNU484 cells treated with SCU when compared with untreated group of cells were grouped based on the enriched pathways using PANTHER database (http://pantherdb.org/). The percentage (\%) of proteins participating in each pathway are shown using a pie chart. GC, gastric cancer; SCU, Scutellarein.

regulation of clathrin-dependent endocytosis, and apoptotic process. VINC is a well-known actin filament binding protein that is involved in the process of cell-cell adhesion, cell-matrix adhesion, and it alters E-cadherin expression on the cell surface. VINC also plays vital roles in cell locomotion and morphology, and downregulation of VINC was found to significantly inhibit pancreatic cancer cell migration (33). Further VINC was also found to stimulate tumor progression by increasing PI3K activation of phosphatidylinositol $(3,4,5)$-triphosphate in cancer cells (34). VCL gene expression was found to be significantly elevated in GC tissues, indicating that VCL may contribute to GC progression by stimulation of tumor malignancy and its invasiveness (35). Targeting the depletion of VCL by anticancer agents may help to overcome cell metastasis and invasion. VINC was downregulated in both GC cell lines treated with SCU, and the biological process of differentially expressed proteins from the current results indicate the involvement of VCL in the locomotion of the cell or its subcellular component and cell projection organization in the SCU-treated GC cells. VAV1, a Dbl superfamily of the Rho/Rac guanine nucleotide exchange factors whose members are predominantly found in the hematopoietic lineage, is well known as a central regulator in the rearrangements of actin cytoskeletal filaments during cell activation. Vav1 acts as a pro-apoptotic factor in breast cancer cells when the expression of p53 is lacking. Studies have targeted VAV1 for its unregulated levels, which could be a strategy to improve breast cancer outcomes by regulating p-Akt (36). VAV1 was elevated in AGS and SNU484 cells treated with SCU, and GO revealed its involvement in the movement of a cell or subcellular component, the regulation of protein kinase B signaling, locomotion and apoptotic process. The current study results of the upregulation of VAV1 may support our previous results that show that SCU induces apoptotic cell death by regulating p53 in GC cells. Pathway analysis showed its involvement in the activation of B cells, and inflammation mediated by cytokine and chemokine signaling pathways, PDGF signaling pathway and activation of $\mathrm{T}$ cells.

BUB1B, DNMT3A, PIK3CA, PDGFRA, DNM1L, ARHGEF2, TLR2, and AKT3 are critical proteins that participate in a majority of biological processes from the $\mathrm{GO}$ analysis of the differentially expressed proteins from SCU-treated GC cells. The mitotic checkpoint serine/threonine-protein kinase that involves kinases (BUB1B) is responsible for chromosome segregation, and plays a key role in spindle checkpoint operation. BUB1B is bound to the kinetochore, and helps inhibition at the anaphase-promoting complex, which tends to procrastinate the initiation of anaphase and provides better chromosome segregation (37). Impaired spindle checkpoint operation has been identified in many forms of cancer. BUB1B is overexpressed and highly correlated with survival time, outperforming markers in the cancer cell, and is a prime therapeutic target in glioblastoma (38). In our study, BUB1B was downregulated by SCU in AGS cells and GO analysis showed its involvement in the apoptotic process. DNA methylation plays a key role in the initiation, as well as the progression of human cancers, providing captivating biomarkers and targets for diagnostic and therapeutic purposes (39). Promoter hypermethylation and deacetylation of tumor-suppressor genes play major roles in cancer induction, through transcriptional silencing of these genes (40). DNA hypermethylation is carried out by a family of DNMTs including DNMT3A. In hepatocellular carcinoma, a positive correlation between the overexpression of these genes and cancer induction has been reported to be significant (41). DNMT3A protein expression was depleted in AGS cells treated with SCU, and functional analysis indicated its role in the apoptotic process by GO. Platelet-derived growth factor receptor $\alpha$ (PDGFRA) encodes a receptor tyrosine kinase (RTK) that activates key pathways such as PI3K/AKT/mTOR that are involved in many types of 
cancers, and it plays a role in several cell functions that include migration, cell proliferation, and angiogenesis (42). Increased PDGFRA expression was found to enhance cell proliferation, migration and reduce apoptosis by the downregulation of miR-140-5p in ovarian cancer (43). The present study result revealed that downregulation of PDGFRA protein expression was observed in SCU-treated AGS cells participating in a major biological process regulated by differentially expressed proteins such as positive regulation of protein kinase B signaling, platelet activation, apoptotic process, and pathways including angiogenesis and the PDGF signaling pathway. Rho guanine exchange factor (ARHGEF2) a microtubule-associated Dbl family member of guanine exchange factors with distinct transaction activity for RHOA, provides cell growth and survival in RAS-transformed cells. ARHGEF2 promotes cell motility via the activation of RhoA signaling in hepatocellular carcinoma in an amplified condition (44). The current results indicate that SCU treatment downregulated the ARHGEF2 protein expression in AGS cells, and ARHGEF2 was found to participate in biological processes, such as cell movement or subcellular component, locomotion, and apoptotic process. Toll-like receptor 2 (TLR2) is a major regulator of the responses of the innate immune system, and alteration of this protein leads to inflammation-related malignancies in $\mathrm{GC}$, where the expression levels at both the mRNA and protein are elevated by more than $50 \%$ in GC patient tumors. The TLR2-promoted growth receptivity of human GC cells coincides with the elevation of anti-apoptotic proteins, such as BCL2, BCL2A1, TNFAIP3, BIRC3, CFLAR, and IER3 (45). Expression of TLR2 and TLR4 is elevated in H.pylori-positive gastritis patients, and their gene polymorphisms are correlated with an elevated risk of GC; thus selectively blocking TLR2 may be a therapeutic approach to the suppression of tumorigenesis (46). SCU treatment showed depletion of TLR2 protein expression in AGS cells, and GO analysis revealed it to participate in biological processes, such as response to macrophage colony-stimulating factor and apoptotic process. AKT3 is a member of the AKT family proteins, and a central protein for the signal mediation from receptor tyrosine kinases, and phosphatidylinositol 3-kinase (PI3K). AKT3 regulates multiple biological processes, such as cell cycle progression, cell proliferation, apoptosis, migration, and invasion (47). Knockdown of AKT3 was found to induce apoptosis by activating caspase- 9 and caspase- 3 in human glioblastoma cell lines U87MG and T98G (48). AKT3 was found to be upregulated in GC tissues compared to that in para-carcinoma tissues at the mRNA level, whereas suppression of AKT3 expression partially by miR-582-5p suppressed tumorigenesis of GC, by promoting cell apoptosis and G0/G1 arrest (49). SCU downregulated the expression of AKT3 in AGS cells, which may support our previous results that demonstrated that activation of caspase- 9 and caspase- 3 in GC cells leads to apoptosis (16). GO analysis of differentially expressed proteins from AGS cells treated with SCU also showed the involvement of AKT3 in biological processes, including cell movement or subcellular component and locomotion, and pathway analysis showed that it participates in angiogenesis, the EGF receptor signaling and apoptosis signaling pathways, exerts hypoxia via HIF activation, participates in the p53 pathway, inflammation mediated by the chemokine and cytokine signaling pathway and PI3 kinase pathway. Apart from all these proteins, another protein that gained our attention which is differentially expressed in SNU484 cells treated with SCU, is CIP2A (cancerous inhibitor of protein phosphatase $2 \mathrm{~A}$ ). As confirmed by immunoblotting, CIP2A protein expression was downregulated after the treatment with SCU in AGS and SNU484 cells. CIP2A gained our attention, due to similar results observed in our previous study where the flavonoid, Pectolinarigenin significantly decreased the protein expression of CIP2A in AGS and MKN28 human GC cells. CIP2A is an oncoprotein that affects cancer cell proliferation, anchorage-independent cell growth, and apoptotic resistance in human cancer cells (26). The inactivation of protein phosphatase 2A (PP2A) occurs by CIP2A depletion and also the phosphorylation of Akt which sustains c-Myc oncogene product in cancer cells. CIP2A has been identified to be elevated at both mRNA and protein levels in GC tissues. Gene silencing approach of CIP2A protein in GC cells was found to reduce the colony-forming potential and proliferation rate of cancer cells, which is a great strategy for the therapy of GC (50). Research has revealed that pharmacological decrease of CIP2A resulted in inhibition of cell proliferation and induced apoptosis in breast cancer cells (51).

Study limitations and further study. The present study consists of the preliminary data of altered proteins upon treatment of GC cells with SCU; in-vivo or solid tumor data were included. In the present study we have selected to validate a subset of critical proteins depending upon the correlation to the current and to our previous study using western blot analysis, and further validation of remaining critical proteins will help to understand the molecular mechanism. Further experiments, such as data using cell lines in vivo with knockdown and/or overexpression experimental models of these biomarker candidates will be conducted, to narrow down the valid biomarker candidate proteins for the treatment of SCU in GC.

In conclusion, the current study highlights an innovative strategy to investigate physiologically relevant targets of SCU treatment, with an emphasis on its functional effects. The synergistic analyses of proteomics data were successful in identifying a unique set of proteins regulated by SCU to induce apoptosis in both treated GC cell lines. Proteins such as PIK3CB, OFD1, CIP2A, BUB1B, ARHGEF2, VCL, EPHB2, IF4G2, CACNA2D1, HIP1R, VAV, and TLR2 have been previously reported to be associated with apoptosis, cell cycle arrest and tumor suppressors induced by another flavonoid in GC cell lines (17). Previous and current data showed that these protein candidates can be altered upon treatment with flavonoids in GC cells. We can consider these proteins for further investigation for their applicability as treatment markers of apoptosis, especially in the context of evaluating the intensity to which GC cells have undergone apoptosis following chemotherapy using flavonoids. In this aspect, the current results may be of clinical utility in the estimation of the GC response to flavonoids, and may aid in their future development as novel clinical therapeutic agents with which to treat GC.

\section{Acknowledgements}

Not applicable. 


\section{Funding}

The present study was supported by the National Research Foundation of Korea funded by the Ministry of Science and ICT (grant nos. 2012M3A9B8019303 and 2020R1A2B5B01001807).

\section{Availability of data and materials}

All data generated or analyzed during this study are included in this published article.

\section{Authors' contributions}

VVGS and GSK conceived and designed the experiments, performed the experiments, organized focus group discussion, collected, analyzed all study data and prepared the final manuscript. PV, RM and HJL carried out the bioinformatics analysis, contributed to the statistical analysis and editing of the manuscript. SMK, SEH, JDH and EHK participated in focus group discussion and revised the study design, revised the results and final revision of the manuscript for publication. All authors read and approved the manuscript for publication.

\section{Ethics approval and consent to participate}

Not applicable.

\section{Patient consent for publication}

Not applicable.

\section{Competing interests}

The authors declare that they have no competing interests.

\section{References}

1. Shin A, Kim J and Park S: Gastric cancer epidemiology in Korea. J Gastric Cancer 11: 135-140, 2011.

2. Rivas-Ortiz CI, Lopez-Vidal Y, Arredondo-Hernandez LJR and Castillo-Rojas G: Genetic alterations in gastric cancer associated with helicobacter pylori infection. Front Med (Lausanne) 4: 47, 2017.

3. Ilson DH: Advances in the treatment of gastric cancer. Curr Opin Gastroenterol 34: 465-468, 2018.

4. Wang S and Yuan L: Predictive biomarkers for targeted and cytotoxic agents in gastric cancer for personalized medicine. Biosci Trends 10: 171-180, 2016.

5. An W, Lai H, Zhang Y, Liu M, Lin X and Cao S: Apoptotic pathway as the therapeutic target for anticancer traditional chinese medicines. Front Pharmacol 10: 758, 2019.

6. Pavet V, Portal MM, Moulin JC, Herbrecht R and Gronemeyer H: Towards novel paradigms for cancer therapy. Oncogene 30: 1-20, 2011.

7. Alessandro R, Fontana S, Kohn E and De Leo G: Proteomic strategies and their application in cancer research. Tumori 91 447-455, 2005.

8. Ludwig JA and Weinstein JN: Biomarkers in cancer staging, prognosis and treatment selection. Nat Rev Cancer 5: 845-856, 2005.

9. Hanash SM, Madoz-Gurpide J and Misek DE: Identification of novel targets for cancer therapy using expression proteomics. Leukemia 16: 478-485, 2002.

10. Yang J, Chen L, Kong X, Huang T and Cai YD: Analysis of tumor suppressor genes based on gene ontology and the KEGG pathway. PLoS One 9: e107202, 2014.
11. Lee HH,Lim CA, Cheong YT, Singh M and Gam LH: Comparison of protein expression profiles of different stages of lymph nodes metastasis in breast cancer. Int J Biol Sci 8: 353-362, 2012.

12. Agbarya A, Ruimi N, Epelbaum R, Ben-Arye E and Mahajna J: Natural products as potential cancer therapy enhancers: A preclinical update. SAGE Open Med 2: 2050312114546924, 2014.

13. Chahar MK, Sharma N, Dobhal MP and Joshi YC: Flavonoids: A versatile source of anticancer drugs. Pharmacogn Rev 5: 1-12, 2011.

14. Lee HJ, Venkatarame Gowda Saralamma V, Kim SM, Ha SE, Raha S, Lee WS, Kim EH, Lee SJ, Heo JD and Kim GS: Pectolinarigenin induced cell cycle arrest, autophagy, and apoptosis in gastric cancer cell via PI3K/AKT/mTOR signaling pathway. Nutrients 10: 1043, 2018.

15. Sang Eun H, Seong Min K, Ho Jeong L, Vetrivel P, Venkatarame Gowda Saralamma V, Jeong Doo H, Eun Hee K, Sang Joon L and Gon Sup K: Scutellarein induces fas-mediated extrinsic apoptosis and G2/M cell cycle arrest in Hep3B hepatocellular carcinoma cells. Nutrients 11: 263, 2019.

16. Gowda Saralamma VV, Lee HJ, Raha S, Lee WS, Kim EH, Lee SJ, Heo JD, Won C, Kang CK and Kim GS: Inhibition of IAP's and activation of $\mathrm{p} 53$ leads to caspase-dependent apoptosis in gastric cancer cells treated with scutellarein. Oncotarget 9: 5993-6006, 2017.

17. Lee HJ, Venkatarame Gowda Saralamma V, Kim SM, Ha SE, Vetrivel P, Kim EH, Lee SJ, Heo JD, Rampogu S, Lee KW and Kim GS: Comparative proteomic profiling of tumor-associated proteins in human gastric cancer cells treated with pectolinarigenin. Nutrients 10: 1596, 2018.

18. Swain M and Ross NW: A silver stain protocol for proteins yielding high resolution and transparent background in sodium dodecyl sulfate-polyacrylamide gels. Electrophoresis 16: 948-951, 1995.

19. Shevchenko A, Wilm M, Vorm O and Mann M: Mass spectrometric sequencing of proteins silver-stained polyacrylamide gels. Anal Chem 68: 850-858, 1996.

20. Baker PR and Chalkley RJ: MS-viewer: A web-based spectral viewer for proteomics results. Mol Cell Proteomics 13: 1392-1396, 2014.

21. Halgren TA, Murphy RB, Friesner RA, Beard HS, Frye LL, Pollard WT and Banks JL: Glide: A new approach for rapid, accurate docking and scoring. 2. Enrichment factors in database screening. J Med Chem 47: 1750-1759, 2004.

22. Tabas-Madrid D, Nogales-Cadenas R and Pascual-Montano A: GeneCodis3: A non-redundant and modular enrichment analysis tool for functional genomics. Nucleic Acids Res 40: W478-W483, 2012.

23. Thomas PD, Campbell MJ, Kejariwal A, Mi H, Karlak B, Daverman R, Diemer K, Muruganujan A and Narechania A: PANTHER: A library of protein families and subfamilies indexed by function. Genome Res 13: 2129-2141, 2003.

24. Szklarczyk D, Gable AL, Lyon D, Junge A, Wyder S, Huerta-Cepas J, Simonovic M, Doncheva NT, Morris JH, Bork P, et al: STRING v11: Protein-protein association networks with increased coverage, supporting functional discovery in genome-wide experimental datasets. Nucleic Acids Res 47: D607-D613, 2019.

25. Starovoytov ON, Liu Y, Tan L and Yang S: Effects of the hydroxyl group on phenyl based ligand/ERR $\gamma$ protein binding. Chem Res Toxicol 27: 1371-1379, 2014.

26. Chen KF, Liu CY, Lin YC, Yu HC, Liu TH, Hou DR, Chen PJ and Cheng AL: CIP2A mediates effects of bortezomib on phospho-Akt and apoptosis in hepatocellular carcinoma cells. Oncogene 29: 6257-6266, 2010.

27. Gao F, Xu T, Wang X, Zhong S, Chen S, Zhang M, Zhang X, Shen Y, Wang X, Xu C and Shen Z: CIP2A mediates fibronectin-induced bladder cancer cell proliferation by stabilizing beta-catenin. J Exp Clin Cancer Res 36: 70, 2017.

28. Feather SA, Woolf AS, Donnai D, Malcolm S and Winter RM: The oral-facial-digital syndrome type 1 (OFD1), a cause of polycystic kidney disease and associated malformations, maps to Xp22.2-Xp22.3. Hum Mol Genet 6: 1163-1167, 1997.

29. Wee S, Wiederschain D, Maira SM, Loo A, Miller C, deBeaumont R, Stegmeier F, Yao YM and Lengauer C: PTEN-deficient cancers depend on PIK3CB. Proc Natl Acad Sci USA 105: 13057-13062, 2008.

30. Riquelme I, Tapia O, Leal P, Sandoval A, Varga MG, Letelier P, Buchegger K, Bizama C, Espinoza JA and Peek RM: miR-101-2, miR-125b-2 and miR-451a act as potential tumor suppressors in gastric cancer through regulation of the PI3K/AKT/mTOR pathway. Cell Oncol (Dordr) 39: 23-33, 2016. 
31. Martini M, De Santis MC, Braccini L, Gulluni F and Hirsch E: PI3K/AKT signaling pathway and cancer: An updated review. Ann Med 46: 372-383, 2014.

32. Rice MA, Ishteiwy RA, Magani F, Udayakumar T, Reiner T, Yates TJ, Miller P, Perez-Stable C, Rai P, Verdun R, et al: The microRNA-23b/-27b cluster suppresses prostate cancer metastasis via Huntingtin-interacting protein 1-related. Oncogene 35: $4752-4761,2016$

33. Ai J, Jin T, Yang L, Wei Q, Yang Y, Li H and Zhu Y: Vinculin and filamin-C are two potential prognostic biomarkers and therapeutic targets for prostate cancer cell migration. Oncotarget 8: 82430-82436, 2017.

34. Rubashkin MG, Cassereau L, Bainer R, DuFort CC, Yui Y, Ou G, Paszek MJ, Davidson MW, Chen YY and Weaver VM: Force engages vinculin and promotes tumor progression by enhancing PI3K activation of phosphatidylinositol $(3,4,5)$-triphosphate. Cancer Res 74: 4597-4611, 2014.

35. Liu W, Zhao Y and Wu J: Gene expression profile analysis of the progression of carotid atherosclerotic plaques. Mol Med Rep 17: 5789-5795, 2018

36. Grassilli S, Brugnoli F, Lattanzio R, Marchisio M, Perracchio L, Piantelli M, Bavelloni A, Capitani S and Bertagnolo V: Vav1 downmodulates Akt in different breast cancer subtypes: A new promising chance to improve breast cancer outcome. Mol Oncol 12: 1012-1025, 2018.

37. Davenport JW, Fernandes ER, Harris LD, Neale GA and Goorha R: The mouse mitotic checkpoint gene bublb, a novel bub1 family member, is expressed in a cell cycle-dependent manner. Genomics 55: 113-117, 1999.

38. Ma Q, Liu Y, Shang L, Yu J and Qu Q: The FOXM1/BUB1B signaling pathway is essential for the tumorigenicity and radioresistance of glioblastoma. Oncol Rep 38: 3367-3375, 2017.

39. Fort L, Batista JM, Thomason PA, Spence HJ, Whitelaw JA, Tweedy L, Greaves J, Martin KJ, Anderson KI, Brown P, et al: Fam49/CYRI interacts with Rac1 and locally suppresses protrusions. Nat Cell Biol 20: 1159-1171, 2018.

40. Sakata M, Kitamura YH, Sakuraba K, Goto T, Mizukami H, Saito M, Ishibashi K, Kigawa G, Nemoto H, Sanada Y and Hibi K: Methylation of HACE1 in gastric carcinoma. Anticancer Res 29: 2231-2233, 2009.

41. Sanaei M, Kavoosi F, Roustazadeh A and Golestan F: Effect of genistein in comparison with trichostatin a on reactivation of dnmts genes in hepatocellular carcinoma. J Clin Trans Hepatol 6: 141-146, 2018
42. Ravichandran K and Edelstein CL: Polycystic kidney disease: A case of suppressed autophagy? Semin Nephrol 34: 27-33, 2014

43. Tang Z, Lin MG, Stowe TR, Chen S, Zhu M, Stearns T, Franco B and Zhong Q: Autophagy promotes primary ciliogenesis by removing OFD1 from centriolar satellites. Nature 502: 254-257, 2013.

44. Kent OA, Sandi MJ, Burston HE, Brown KR and Rottapel R: An oncogenic KRAS transcription program activates the RHOGEF ARHGEF2 to mediate transformed phenotypes in pancreatic cancer. Oncotarget 8: 4484-4500, 2017.

45. West AC, Tang K, Tye H, Yu L, Deng N, Najdovska M, Lin SJ, Balic JJ, Okochi-Takada E, McGuirk P, et al: Identification of a TLR2-regulated gene signature associated with tumor cell growth in gastric cancer. Oncogene 36: 5134-5144, 2017.

46. de Oliveira JG and Silva AE: Polymorphisms of the TLR2 and TLR4 genes are associated with risk of gastric cancer in a Brazilian population. World J Gastroenterol 18: 1235-1242, 2012.

47. Li L and Ma L: Upregulation of miR-582-5p regulates cell proliferation and apoptosis by targeting AKT3 in human endometrial carcinoma. Saudi J Biol Sci 25: 965-970, 2018.

48. Turner KM, Sun Y, Ji P, Granberg KJ, Bernard B, Hu L, Cogdell DE, Zhou X, Yli-Harja O, Nykter M, et al: Genomically amplified Akt3 activates DNA repair pathway and promotes glioma progression. Proc Natl Acad Sci USA 112: 3421-3426, 2015.

49. Jin Y, Tao LP, Yao SC, Huang QK, Chen ZF, Sun YJ and Jin SQ: MicroRNA-582-5p suppressed gastric cancer cell proliferation via targeting AKT3. Eur Rev Med Pharmacol Sci 21: 5112-5120, 2017.

50. Li W, Ge Z, Liu C, Liu Z, Björkholm M, Jia J and Xu D: CIP2A is overexpressed in gastric cancer and its depletion leads to impaired clonogenicity, senescence, or differentiation of tumor cells. Clin Cancer Res 14: 3722-3728, 2008.

51. Niemela M, Kauko O, Sihto H, Mpindi JP, Nicorici D, Pernilä $\mathrm{P}$, Kallioniemi OP, Joensuu $\mathrm{H}$, Hautaniemi $\mathrm{S}$ and Westermarck J: CIP2A signature reveals the MYC dependency of CIP2A-regulated phenotypes and its clinical association with breast cancer subtypes. Oncogene 31: 4266-4278, 2012.

This work is licensed under a Creative Commons Attribution-NonCommercial-NoDerivatives 4.0 International (CC BY-NC-ND 4.0) License. 